\title{
Some constructions for the fractional Laplacian on noncompact manifolds
}

\author{
Valeria Banica, María del Mar González and Mariel Sáez
}

\begin{abstract}
We give a definition of the fractional Laplacian on some noncompact manifolds, through an extension problem introduced by Caffarelli-Silvestre. While this definition in the compact case is straightforward, in the noncompact setting one needs to have a precise control of the behavior of the metric at infinity and geometry plays a crucial role. First we give explicit calculations in the hyperbolic space, including a formula for the kernel and a trace Sobolev inequality. Then we consider more general noncompact manifolds, where the problem reduces to obtain suitable upper bounds for the heat kernel.
\end{abstract}

\section{Introduction and statement of the results}

There are extensive works involving fractional order operators. In particular, nonlinear or free boundary problems involving fractional powers of the Laplacian $(-\Delta)^{\gamma}$ appear naturally in applications (see for instance [67], [62] and the references therein). As pseudodifferential operators, the classical definitions involve functional analysis and singular integrals. They are nonlocal objects, which means that a priori estimates and maximum principles are not easy to obtain. However, in the Euclidean case, Caffarelli and Silvestre have developed in [11] an equivalent definition using an extension problem in one more dimension in terms of a degenerate elliptic equation with $\mathcal{A}_{2}$ weight, of the type studied by FabesJerison-Kenig-Serapioni [25], [26].

On the other hand, from the geometry side there is the work of GrahamZworski [33] that studies a general class of conformally covariant operators $P_{\gamma}$ defined on a compact manifold $M^{n}$. These operators are defined through scattering theory [53] when $M$ is the boundary $M^{n}$ of a conformally compact Einstein manifold. In [15] both the geometrical and the PDE points of view were recon-

Mathematics Subject Classification (2010): 26A33, 35J70, 58J40.

Keywords: Fractional Laplacian, non-compact manifolds, hyperbolic space, extension problem, Fourier symbol, singular kernel. 
ciled and, in particular, the fractional Laplacian on the sphere $\mathbb{S}^{n}$ (or $\mathbb{R}^{n}$ through stereographic projection) is defined from scattering theory in the Poincaré ball.

It is possible then to formulate fractional Yamabe-type problems for $P_{\gamma}$, as considered in [31], where the main ingredients needed in the proof are a Hopf's maximum principle, elliptic estimates and a sharp Sobolev trace inequality. These are shown by means of the extension formulation of Caffarelli-Silvestre its generalization on manifolds by [15].

However, if $M^{n}$ is a noncompact manifold with a Riemannian metric $g_{M}$, these methods are not available in general since it is not clear how to define fractional order operators in the noncompact setting. One can give a reasonable definition when $M$ is an open dense set in a compact manifold $\hat{M}$ and the metric $g_{M}$ is conformally related to a smooth metric $\hat{g}$ on $\hat{M}$. Namely, we can define $P_{\gamma}$ by demanding that a conformally covariant relationship holds. Note, however, that this is not as simple as it first appears. In [30] singular fractional Yamabe problems were considered in the particular case that $M=S^{n} \backslash \Lambda$, where the singular set $\Lambda$ is a smooth $k$-dimensional submanifold and $g_{M}$ a complete metric with controlled growth near the singular set. Not much is known in the general noncompact case.

In the present paper we try to formulate an extension problem for the fractional Laplacian $\left(-\Delta_{M}\right)^{\gamma}$ on hyperbolic space and on some other noncompact manifolds. It is a very interesting open question to set up a conformally covariant version of the operator (that has the same principal symbol as $\left.\left(-\Delta_{M}\right)^{\gamma}\right)$; we hope to return to this problem elsewhere.

More precisely, we will give sufficient conditions on the underlying manifold for the following to hold:

Theorem 1.1. Let $M^{n}$ be a n-dimensional complete, non-compact manifold with a Riemannian metric $g_{M}$ satisfying any of the conditions in Proposition 3.3, which in particular include hyperbolic space $\mathbb{H}^{n}$. Let $g$ be the product metric on $M \times \mathbb{R}_{+}$ given by $g=g_{M}+d y^{2}$. Set $\gamma \in(0,1)$ and $a=1-2 \gamma$. For any given $f \in H^{\gamma}(M)$, there exists a unique solution of the extension problem

$$
\left\{\begin{aligned}
\operatorname{div}_{g}\left(y^{a} \nabla_{g} u\right)(x, y) & =0 & & \text { for } \quad(x, y) \in M \times \mathbb{R}_{+}, \\
u(x, 0) & =f(x) & & \text { for } x \in M .
\end{aligned}\right.
$$

Moreover, the fractional Laplacian on $M$ is well defined and can be recovered through

$$
\left(-\Delta_{M}\right)^{\gamma} f=-d_{\gamma} \lim _{y \rightarrow 0} y^{a} \partial_{y} u
$$

for a constant

$$
d_{\gamma}=2^{2 \gamma-1} \frac{\Gamma(\gamma)}{\Gamma(1-\gamma)}
$$

In view of the definition of the metric $g$, the extension problem (1.1) writes

$$
\left\{\begin{aligned}
\partial_{y y} v+\frac{a}{y} \partial_{y} v+\Delta_{M} v(x, y) & =0 & & \text { for } \quad(x, y) \in M \times \mathbb{R}_{+}, \\
v(x, 0) & =f(x) & & \text { for } x \in M .
\end{aligned}\right.
$$


In the case of hyperbolic space one is able to carry out very explicit calculations, since Fourier analysis and harmonic analysis tools are available. Indeed, $\mathbb{H}^{n}$ is the simplest example of a symmetric space of rank one. In addition, we give a precise formula for $\left(-\Delta_{\mathbb{H}^{n}}\right)^{\gamma}$ in terms of a singular integral obtained as convolution with a well behaved kernel (see Theorem 2.5). Elliptic a priori estimates may be obtained by understanding the asymptotics of this kernel; we show Hölder estimates as an application.

These results allow to set up semilinear problems for the fractional Laplacian on hyperbolic space. When the nonlinearity comes from a double well potential, one has existence and uniqueness of layer solutions, as well as some symmetry results (see [32]).

Semilinear equations for the usual Laplace-Beltrami operator on hyperbolic space were studied in [51], [13], for instance, in relation to conformal geometry. In their works, a Sobolev inequality for the hyperbolic Laplacian appears naturally [52]. In [41], the authors study the Paneitz operator on hyperbolic space, that is a conformally covariant operator of order 4. Higher order Sobolev inequalities were considered in [50].

It is still an open question to set up the conformal geometry interpretation of the hyperbolic fractional Laplacian, and to study the associated fractional Yamabe problems. One first step is to obtain trace Sobolev embeddings. This is the content of Theorem 2.9. The key idea, as in the Euclidean case, is to study the energy associated to problem (1.4), which allows to obtain sharp inequalities. Note that the best constant in this embedding is related to the Yamabe constant in the model case for the fractional Yamabe problem.

From the probability point of view, see the survey [17] for a construction of fractional Lévy Brownian fields on hyperbolic space.

We note here that the fractional Laplacian on the torus constructed from the extension (1.1) has been considered in [58], using a double Fourier series expansion. However, as we have mentioned, the question on noncompact manifolds, where Fourier analysis is not available, is more delicate and not much is known. Here we try to give a first approach.

The relation between heat kernel and fractional powers of an operator is a very old one. From the spectral theory and functional calculus point of view, Stinga and Torrea [62] show that one can define the fractional Laplacian on a domain $\Omega \subset \mathbb{R}^{n}$ through the extension (1.1) provided that the heat kernel associated to $-\Delta_{\Omega}$ exists and it satisfies some decay properties. Since the heat kernel on general noncompact manifolds has been extensively studied depending on the underlying geometry, we take this approach to prove Theorem 1.1.

The organization of the paper is as follows: In Section 2, based on the results in $\mathbb{R}^{n}$, we concentrate on definitions and properties of the fractional Laplacian on hyperbolic space. We include a definition in terms of the Fourier transform (Subsection 2.2), an expression in terms of a singular integral (Theorem 2.5 and its proof in Subsection 2.3.1) and the relation with an appropriate extension problem (both in terms of a Poisson kernel and and energy formulation, in Subsections 2.3.1 and 2.3.2 respectively). These results imply regularity, a Hopf's maximum prin- 
ciple and sharp trace Sobolev inequality. We finish Section 2 by discussing how to extend the previous results to other harmonic groups (Subsection 2.4). In Section 3 we start by discussing a general framework under which the results in [62] can be generalized to non-compact manifolds. In order to carry the construction of a fractional Laplacian, it is necessary to obtain bounds on the heat kernel. These bounds are discussed in Subsection 3.2. In the following subsections we workout examples of manifolds that fulfill the required conditions. Among these are symmetric spaces, some geometrically finite hyperbolic manifolds, a class of rotationally symmetric manifolds and certain manifolds with ends.

Acknowledgements. The authors would like to thank Thierry Couhlon for useful remarks on $\S 3.2$, Carlo Morpurgo, and the anonymous referee.

\section{The extension problem on hyperbolic space}

The real hyperbolic space $\mathbb{H}^{n}, n \geq 2$, is the simplest example of Riemannian symmetric spaces of the noncompact type. Fourier analysis on (noncompact) Riemannian symmetric spaces has been well studied. We refer to Helgason's books [44], [46], [45] for the basic Fourier theory, to [2] for the theory of $L^{p}$ multipliers and to [4] for heat kernel and Green function estimates.

\subsection{Notations and definitions}

Several models for the $n$-dimensional hyperbolic space $\mathbb{H}^{n}$ have been considered in the literature. Here we will define it as the upper branch of a hyperboloid in $\mathbb{R}^{n+1}$ with the metric induced by the Lorentzian metric in $\mathbb{R}^{n+1}$ given by $-d x_{0}^{2}+d x_{1}^{2}+$ $\cdots+d x_{n}^{2}$. More precisely, we take

$$
\begin{aligned}
\mathbb{H}^{n} & =\left\{\left(x_{0}, \ldots, x_{n}\right) \in \mathbb{R}^{n+1}: x_{0}^{2}-x_{1}^{2}-\cdots-x_{n}^{2}=1, x_{0}>0\right\} \\
& =\left\{x \in \mathbb{R}^{n+1}: x=(\cosh r, \sinh r \omega), r \geq 0, \omega \in \mathbb{S}^{n-1}\right\},
\end{aligned}
$$

with the metric

$$
g_{\mathbb{H} n}=d r^{2}+\sinh ^{2} r d \omega^{2},
$$

where $d \omega^{2}$ is the metric on $\mathbb{S}^{n-1}$. Under these definitions the Laplace-Beltrami operator is given by

$$
\Delta_{\mathbb{H} n}=\partial_{r r}+(n-1) \frac{\cosh r}{\sinh r} \partial_{r}+\frac{1}{\sinh ^{2} r} \Delta_{\mathbb{S}^{n-1}},
$$

and the volume element is

$$
\sinh ^{n-1} r d r d \omega .
$$

We denote by $[\cdot, \cdot]$ the internal product induced by the Lorentzian metric

$$
\left[x, x^{\prime}\right]=x_{0} x_{0}^{\prime}-x_{1} x_{1}^{\prime}-\cdots-x_{n} x_{n}^{\prime} .
$$

The hyperbolic space is invariant under $S O(1, n)$, the group of Lorentz transformations of $\mathbb{R}^{n+1}$ that preserve this inner product. $\mathbb{H}^{n}$ can be actually defied as 
the quotient between the orbit $S O(1, n) e_{0}$ of the origin $e_{0}=(1,0, \ldots, 0)$ by the stabilizer of $e_{0}$, so that

$$
\mathbb{H}^{n} \approx \frac{S O(1, n)}{S O(n)} .
$$

In particular, using Cartan's decomposition, hyperboloids are symmetric spaces of rank one. Finally, let us recall that, by means of stereographic projection through the hyperboloid origin, Poincaré's disk model is recovered, and from the disk model one obtains the model of the upper half-space by performing an inversion in a boundary point of the ball.

\subsection{Fourier transform and the fractional Laplacian}

We start by reviewing some basic facts about Fourier transform on hyperbolic space, see [29], [44] and [64].

Recall first that in $\mathbb{R}^{n}$ the Fourier transform is given by

$$
\hat{f}(\xi)=\int_{\mathbb{R}^{n}} f(x) e^{-i x \cdot \xi} d x .
$$

Notice that the functions $e^{-i x \cdot \xi}$ are generalized (in the sense that they do not belong to $L^{2}$ ) eigenfunctions of the Laplacian associated to the eigenvalue $-|\xi|^{2}$. Moreover, the following inversion formula holds:

$$
f(x)=\frac{1}{(2 \pi)^{n}} \int_{\mathbb{R}^{n}} \hat{f}(\xi) e^{i x \cdot \xi} d \xi .
$$

Similarly, in $\mathbb{H}^{n}$ we consider the generalized eigenfunctions of the LaplaceBeltrami operator:

$$
h_{\lambda, \theta}(x)=[x,(1, \theta)]^{i \lambda-(n-1) / 2}, \quad x \in \mathbb{H}^{n},
$$

where $\lambda \in \mathbb{R}$ and $\theta \in \mathbb{S}^{n-1}$, that satisfy

$$
\Delta_{\mathbb{H}^{n}} h_{\lambda, \theta}=-\left(\lambda^{2}+\frac{(n-1)^{2}}{4}\right) h_{\lambda, \theta} .
$$

In analogy with the Euclidean setting, the Fourier transform can be defined as (see Chapter III, equation (4) in [44] or Chapter 3, equation 3.5 in [29]),

$$
\hat{f}(\lambda, \theta)=\int_{\mathbb{H}^{n}} f(x) h_{\lambda, \theta}(x) d x,
$$

for $\lambda \in \mathbb{R}, \omega \in \mathbb{S}^{n-1}$, where $d x$ is the volume element in hyperbolic space. Moreover, the following inversion formula holds:

$$
f(x)=\int_{-\infty}^{\infty} \int_{\mathbb{S}^{n-1}} \bar{h}_{\lambda, \theta}(x) \hat{f}(\lambda, \theta) \frac{d \theta d \lambda}{|c(\lambda)|^{2}},
$$

where $c(\lambda)$ is the Harish-Chandra coefficient:

$$
\frac{1}{|c(\lambda)|^{2}}=\frac{1}{2(2 \pi)^{n}} \frac{\left|\Gamma\left(i \lambda+\frac{n-1}{2}\right)\right|^{2}}{|\Gamma(i \lambda)|^{2}} .
$$


Similarly, Plancherel formula holds:

$$
\int_{\mathbb{H}^{n}}|f(x)|^{2} d x=\int_{\mathbb{R} \times \mathbb{S}^{n-1}}|\hat{f}(\lambda, \theta)|^{2} \frac{d \theta d \lambda}{|c(\lambda)|^{2}} .
$$

It is easy to check by integration by parts for compactly supported functions (and consequently, for every $f \in L^{2}\left(\mathbb{H}^{n}\right)$ ) that

$$
\begin{aligned}
\widehat{\Delta_{\mathbb{H}^{n}} f}(\lambda, \theta) & =\int_{\mathbb{H}^{n}} \Delta_{\mathbb{H}^{n}} f(x) h_{\lambda, \theta}(x) d x=\int_{\mathbb{H}^{n}} f(x) \Delta_{\mathbb{H}^{n}} h_{\lambda, \theta}(x) d x \\
& =-\left(\lambda^{2}+\frac{(n-1)^{2}}{4}\right) \hat{f}(\lambda, \theta) .
\end{aligned}
$$

Having in mind the theory of spherically symmetric multipliers, we define the fractional Laplacian on hyperbolic space $\left(-\Delta_{\mathbb{H}^{n}}\right)^{\gamma} f$ as the operator that satisfies

$$
\left(-\widehat{\left.\Delta_{\mathbb{H} n}\right)^{\gamma}} f=\left(\lambda^{2}+\frac{(n-1)^{2}}{4}\right)^{\gamma} \hat{f} .\right.
$$

Due to the inversion formula we could write

$$
\begin{aligned}
\left(-\Delta_{\mathbb{H}^{n}}\right)^{\gamma} f(x) & =\int_{-\infty}^{\infty} \int_{\mathbb{S}^{n-1}} \bar{h}_{\lambda, \theta}(x)\left(\lambda^{2}+\frac{(n-1)^{2}}{4}\right)^{\gamma} \hat{f}(\lambda, \theta) \frac{d \theta d \lambda}{|c(\lambda)|^{2}} \\
& =\int_{-\infty}^{\infty}\left[\int_{\mathbb{H}^{n}}\left(\lambda^{2}+\frac{(n-1)^{2}}{4}\right)^{\gamma} k_{\lambda}\left(x, x^{\prime}\right) f\left(x^{\prime}\right) d x^{\prime}\right] d \lambda
\end{aligned}
$$

with

$$
k_{\lambda}\left(x, x^{\prime}\right)=\frac{1}{|c(\lambda)|^{2}} \int_{\mathbb{S}^{n-1}} \bar{h}_{\lambda, \theta}(x) h_{\lambda, \theta}\left(x^{\prime}\right) d \theta .
$$

Since the Laplacian commutes with the action of $g \in S O(1, n)$ we have that

$$
k_{\lambda}\left(x, x^{\prime}\right)=k_{\lambda}\left(g x, g x^{\prime}\right),
$$

and in particular,

$$
k_{\lambda}\left(x, x^{\prime}\right)=k_{\lambda}\left(d_{\mathbb{H}^{n}}\left(x, x^{\prime}\right)\right) .
$$

Denote $\rho=d_{\mathbb{H}^{n}}\left(x, x^{\prime}\right)$. It is natural to define the singular kernel

$$
\mathcal{K}_{\gamma}(\rho)=\int_{-\infty}^{\infty}\left(\lambda^{2}+\frac{(n-1)^{2}}{4}\right)^{\gamma} k_{\lambda}(\rho) d \lambda,
$$

and to (formally) calculate

$$
\left(-\Delta_{\mathbb{H}^{n}}\right)^{\gamma} f(x)=\int_{\mathbb{H}^{n}} f\left(x^{\prime}\right) \mathcal{K}_{\gamma}\left(d_{\mathbb{H}^{n}}\left(x, x^{\prime}\right)\right) d x^{\prime} .
$$

Note that, by the invariance of the problem by $S O(1, n)$, we may rewrite the singular integral as follows: let $g_{x}$ be a transformation that takes 0 into $x$, and 
change variables $x^{\prime}=g_{x} \tilde{x}$. Since $g_{x}$ is an isometry, $\rho=d_{\mathbb{H}^{n}}\left(x^{\prime}, x\right)=d_{\mathbb{H}^{n}}(\tilde{x}, 0)$. Then

$$
\left(-\Delta_{\mathbb{H}^{n}}\right)^{\gamma} f(x)=\int_{\mathbb{H}^{n}} f\left(g_{x} \tilde{x}\right) \mathcal{K}_{\gamma}\left(d_{\mathbb{H}^{n}}(\tilde{x}, 0)\right) d \tilde{x}
$$

In the following we aim to make this formulation precise. For that, we need to recall the following formulas for $k_{\lambda}$ (see, for instance, [7]; one can easily obtain the constants in Lemmas 3.2-3.3 of [7]), where by $\partial_{\rho} / \sinh \rho$ we mean first the derivative operator $\partial_{\rho}$, and then the multiplication by $1 / \sinh \rho$.

Lemma 2.1. We have that

$$
k_{\lambda}(\rho)=(-1)^{(n-1) / 2} 2^{-(n+1) / 2} \pi^{-(n+1) / 2}\left(\frac{\partial_{\rho}}{\sinh \rho}\right)^{(n-1) / 2}(\cos \lambda \rho)
$$

for $n \geq 3$ odd, and for $n \geq 2$ even,

$$
k_{\lambda}(\rho)=(-1)^{n / 2} 2^{-(n+1) / 2} \pi^{-(n+2) / 2} \int_{\rho}^{\infty} \frac{\sinh r}{\sqrt{\cosh r-\cosh \rho}}\left(\frac{\partial_{r}}{\sinh r}\right)^{n / 2}(\cos \lambda r) d r
$$

Remark 2.2. Note that $k_{\gamma}(\rho)$ is an even function, and so is $\mathcal{K}_{\gamma}(\rho)$ from $(2.3)$.

We will also need this well known result (see [1]):

Lemma 2.3. The solution of the ODE

$$
\partial_{s s} \varphi+\frac{\alpha}{s} \partial_{s} \varphi-\varphi=0
$$

may be written as $\varphi(s)=s^{\nu} \psi(s)$, for $\alpha=1-2 \nu$, where $\psi$ solves the the well-known Bessel equation

$$
s^{2} \psi^{\prime \prime}+s \psi^{\prime}-\left(s^{2}+\nu^{2}\right) \psi=0 .
$$

In addition, (2.7) has two linearly independent solutions, $I_{\nu}$ and $K_{\nu}$, which are the modified Bessel functions; their asymptotic behavior is given precisely by

$$
\begin{aligned}
I_{\nu}(s) \sim & \frac{1}{\Gamma(\nu+1)}\left(\frac{s}{2}\right)^{\nu}\left(1+\frac{s^{2}}{4(\nu+1)}+\frac{s^{4}}{32(\nu+1)(\nu+2)}+\cdots\right), \\
K_{\nu}(s) \sim & \frac{\Gamma(\nu)}{2}\left(\frac{2}{s}\right)^{\nu}\left(1+\frac{s^{2}}{4(1-\nu)}+\frac{s^{4}}{32(1-\nu)(2-\nu)}+\cdots\right) \\
& +\frac{\Gamma(-\nu)}{2}\left(\frac{s}{2}\right)^{\nu}\left(1+\frac{s^{2}}{4(\nu+1)}+\frac{s^{4}}{32(\nu+1)(\nu+2)}+\cdots\right),
\end{aligned}
$$

for $s \rightarrow 0^{+}, \nu \notin \mathbb{Z}$. And when $s \rightarrow+\infty$,

$$
\begin{aligned}
I_{\nu}(s) & \sim \frac{1}{\sqrt{2 \pi s}} e^{s}\left(1-\frac{4 \nu^{2}-1}{8 s}+\frac{\left(4 \nu^{2}-1\right)\left(4 \nu^{2}-9\right)}{2 !(8 s)^{2}}-\cdots\right), \\
K_{\nu}(s) & \sim \sqrt{\frac{\pi}{2 s}} e^{-s}\left(1+\frac{4 \nu^{2}-1}{8 s}+\frac{\left(4 \nu^{2}-1\right)\left(4 \nu^{2}-9\right)}{2 !(8 s)^{2}}+\cdots\right) .
\end{aligned}
$$


We now look at the asymptotics of the kernel defined in (2.3):

Theorem 2.4. For $\gamma \in(-1 / 2,1)$ it holds that

- For $n \geq 3$ odd,

$$
\mathcal{K}_{\gamma}(\rho)=\alpha_{\gamma}\left(\frac{\partial_{\rho}}{\sinh \rho}\right)^{(n-1) / 2} \rho^{-1 / 2-\gamma} K_{-1 / 2-\gamma}\left(\frac{n-1}{2} \rho\right) .
$$

- When $n \geq 2$ is even,

$$
\mathcal{K}_{\gamma}(\rho)=\alpha_{\gamma}^{\prime} \int_{\rho}^{\infty} \frac{\sinh r}{\sqrt{\cosh r-\cosh \rho}}\left(\frac{\partial_{r}}{\sinh r}\right)^{n / 2}\left[r^{-1 / 2-\gamma} K_{-1 / 2-\gamma}\left(\frac{n-1}{2} r\right)\right] d r .
$$

Here $K_{-1 / 2-\gamma}$ is the solution to the modified Bessel equation given by Lemma 2.3. Additionally, $\mathcal{K}_{\gamma}(\rho)$ has the asymptotic behavior:

(i) $A s \rho \rightarrow 0$,

$$
\mathcal{K}_{\gamma}(\rho) \sim \rho^{-n-2 \gamma}
$$

(ii) As $\rho \rightarrow \infty$,

$$
\mathcal{K}_{\gamma}(\rho) \sim \rho^{-1-\gamma} e^{-(n-1) \rho} .
$$

Proof. We assume that $n$ is odd; the calculations for $n$ even are similar. Using (2.5) we have formally for $n \geq 3$ that

$$
\begin{aligned}
\mathcal{K}_{\gamma}(\rho) & =(-1)^{(n-1) / 2}(2 \pi)^{-(n+1) / 2}\left(\frac{\partial_{\rho}}{\sinh \rho}\right)^{(n-1) / 2}\left(\int_{-\infty}^{\infty}\left(\lambda^{2}+\frac{(n-1)^{2}}{4}\right)^{\gamma} \cos \lambda \rho d \lambda\right) \\
& =(-1)^{(n-1) / 2}(2 \pi)^{-(n+1) / 2}\left(\frac{\partial_{\rho}}{\sinh \rho}\right)^{(n-1) / 2}\left(\int_{-\infty}^{\infty}\left(\lambda^{2}+\frac{(n-1)^{2}}{4}\right)^{\gamma} e^{-i \lambda \rho} d \lambda\right) .
\end{aligned}
$$

The last equality follows from $\left(\lambda^{2}+(n-1)^{2} / 4\right)^{\gamma} \sin \lambda \rho$ being odd.

Notice that the equality above holds when $\gamma<-1 / 2$, while for bigger values of $\gamma$ needs to be interpreted in the sense of distributions. Hence, we need to compute first the distributional Fourier transform (in $\mathbb{R}$ ) of

$$
h(\lambda):=\left(\lambda^{2}+\frac{(n-1)^{2}}{4}\right)^{\gamma} .
$$

Since $\left(\lambda^{2}+(n-1)^{2} / 4\right)^{\gamma}$ is a tempered distribution, it has Fourier transform which is also tempered distribution.

Moreover,

$$
\left(\lambda^{2}+\frac{(n-1)^{2}}{4}\right) \partial_{\lambda} h=2 \lambda \gamma h
$$

Taking Fourier transform, we have

$$
\left(-\partial_{\rho \rho}+\frac{(n-1)^{2}}{4}\right)(i \rho \hat{h})=2 \gamma\left(i \partial_{\rho} \hat{h}\right),
$$

or equivalently

$$
\rho \partial_{\rho \rho} \hat{h}+2(1+\gamma) \partial_{\rho} \hat{h}-\frac{(n-1)^{2}}{4} \rho \hat{h}=0
$$


By performing the change of variables $s=\frac{n-1}{2} \rho$ and denoting $\varphi(s)=\hat{h}(\rho)$, we obtain the ODE

$$
\partial_{s s} \varphi+\frac{2(1+\gamma)}{s} \partial_{s} \varphi-\varphi=0 .
$$

From Lemma 2.3 we have that the solution may be written as

$$
\hat{h}(\rho)=\rho^{-1 / 2-\gamma}\left(\tilde{\alpha}_{\gamma} K_{-1 / 2-\gamma}\left(\frac{n-1}{2} \rho\right)+\tilde{\beta}_{\gamma} I_{-1 / 2-\gamma}\left(\frac{n-1}{2} \rho\right)\right),
$$

where $K_{-1 / 2-\gamma}$ and $I_{-1 / 2-\gamma}$ are the solutions to the modified Bessel equation given in the same lemma.

Since $h$ is a tempered distribution, $\hat{h}$ can at most have polynomial growth, hence, necessarily $\tilde{\beta}_{\gamma}=0$. Notice that for $\gamma<0$ the function $\hat{h}$ is integrable near $\rho=0$, while for $\gamma>0$ it will need to be interpreted in the principal value sense (see Theorem 2.5 below). So (2.8) is proved. For getting (2.9) we first apply the operator $\left(\partial_{\rho} / \sinh \rho\right)^{(n-1) / 2}$ on $\hat{h}$ by using Leibniz rule and the recursive relation $\partial_{\rho}\left(\rho^{a} K_{a}(\rho)\right)=\rho\left(\rho^{a-1} K_{a-1}(\rho)\right)$. Then, for the resulting Bessel functions we use the asymptotic expansions from Lemma 2.3 to obtain (2.9).

For $\gamma<0, \mathcal{K}_{\gamma}$ plays the role of the Riesz potential on Euclidean space. As a consequence, because of the asymptotics (2.9), we have that expression (2.4) for the fractional Laplacian is justified when $\gamma<0$. On the other hand, for $\gamma>0$ we have:

Theorem 2.5. Fix $\gamma \in(0,1)$. Let $f \in L^{1}\left(\mathbb{H}^{n}\right) \cap \mathcal{C}^{\alpha}\left(\mathbb{H}^{n}\right)$ for some $\alpha>2 \gamma$. Then

$$
\left(-\Delta_{\mathbb{H}^{n}}\right)^{\gamma} f(x)=P . V . \int_{\mathbb{H}^{n}}\left(f\left(x^{\prime}\right)-f(x)\right) \mathcal{K}_{\gamma}\left(d_{\mathbb{H}^{n}}\left(x^{\prime}, x\right)\right) d x^{\prime} .
$$

The proof will be postponed until next section, where we shall show also that $\mathcal{K}_{\gamma}$ is a positive kernel for $\gamma \in(0,1)$.

There are several ways to define the Sobolev spaces on hyperbolic space and more generally on manifolds. We present some of them and refer to [65] and the references therein. For a given $n$-dim manifold $M$ with positive injectivity radius and bounded geometry the Sobolev spaces $W_{p}^{k}(M)$ with $k$ integer were first defined as

$$
W_{p}^{k}(M)=\left\{f \in L^{p}(M), \nabla_{g}^{l} f \in L^{p}(M), \forall 1 \leq l \leq k\right\}
$$

with norm $\|f\|_{W_{p}^{k}(M)}=\Sigma_{l=0}^{k}\left\|\nabla_{g}^{l} f\right\|_{L^{p}(M)}$.

The fractional spaces $H_{p}^{\gamma}(M)$ with $\gamma>0$ are

$$
H_{p}^{\gamma}(M)=\left\{f \in L^{p}(M), \exists h \in L^{p}(M), f=(i d-\Delta)^{-\gamma / 2} h\right\},
$$

with norm $\|f\|_{H_{p}^{\gamma}(M)}=\|h\|_{L^{p}(M)}$.

A similar definition is given also for $\gamma<0$. Lizorkin-Triebel spaces $F_{p q}^{\gamma}$, that rely on dyadic analysis, were defined on $M$ by the localization principle. The 
Paley-Littlewood theorem makes the links between there three classes of spaces for $1<p<\infty$ :

$$
W_{p}^{k}(M)=H_{p}^{k}(M)=F_{p 2}^{k} \quad \text { for } k \in \mathbb{N}, \quad H_{p}^{\gamma}(M)=F_{p 2}^{\gamma} \quad \text { for } \gamma \in \mathbb{R} .
$$

For $-\infty<\gamma_{1} \leq \gamma_{2}<+\infty$ and $0<p<+\infty$, the following Sobolev embedding holds:

$$
H_{p}^{\gamma_{1}}(M) \subseteq H_{p}^{\gamma_{2}}(M)
$$

Let $\gamma \in \mathbb{R}$ and $p \in(1, \infty)$. For stratified Lie groups or symmetric spaces (see [27] and [2]; see also $§ 3$ of [63] for hyperbolic space), the following equivalence was proved:

$$
H_{p}^{\gamma}(M)=\left\{f \in L^{p}(M),\|f\|_{L^{p}(M)}+\left\|(-\Delta)^{\gamma / 2} f\right\|_{L^{p}(M)}<\infty\right\} .
$$

In our notation we will drop the subindex $p$ in the $p=2$ case.

Finally, the pointwise formula from Theorem 2.5 allows to prove some regularity estimates as the ones in [61]. The following proposition is obtained from (2.10) together with the decay estimates from Theorem 2.4. Since proof is the same one as in the Euclidean case, we refer the reader to [61]. The ingredients needed there are the estimates for the kernel and the Riesz transform (we recall that Riesz transform $R=\nabla \Delta^{-1 / 2}$ can be defined on $\mathbb{H}^{n}$, see [43], [10]).

Proposition 2.6. Let $w=\left(-\Delta_{\mathbb{H} n}\right)^{\gamma} f$.

(a) Let $f \in \mathcal{C}^{k, \alpha}\left(\mathbb{H}^{n}\right)$, and suppose that $k+\alpha-2 \gamma$ is not an integer. Then

$$
w \in \mathcal{C}^{l, \beta},
$$

where $l$ is the integer part of $k+\alpha-2 \gamma$ and $\beta=k+\alpha-2 \gamma-l$.

(b) Assume that, for some $\alpha \in(0,1], w \in \mathcal{C}^{0, \alpha}\left(\mathbb{H}^{n}\right)$ and $f \in L^{\infty}\left(\mathbb{H}^{n}\right)$. Then

(b1) If $\alpha+2 \gamma \leq 1$, then $f \in \mathcal{C}^{0, \alpha+2 \gamma}\left(\mathbb{H}^{n}\right)$. Moreover,

$$
\|f\|_{\mathcal{C}^{0, \alpha+2 \gamma}} \leq C\left(\|f\|_{L^{\infty}}+\|w\|_{\mathcal{C}^{0, \alpha}}\right)
$$

(b2) If $\alpha+2 \gamma>1$, then $f \in \mathcal{C}^{1, \alpha+2 \gamma-1}\left(\mathbb{H}^{n}\right)$. Moreover,

$$
\|f\|_{\mathcal{C}^{1, \alpha+2 \gamma-1}} \leq C\left(\|f\|_{L^{\infty}}+\|w\|_{\mathcal{C}^{0, \alpha}}\right) .
$$

(c) Assume that $w, f \in L^{\infty}\left(\mathbb{H}^{n}\right)$. Then

(c1) If $2 \gamma \leq 1$, then $f \in \mathcal{C}^{0, \alpha}\left(\mathbb{H}^{n}\right)$ for any $\alpha<2 \gamma$. Moreover,

$$
\|f\|_{\mathcal{C}^{0, \alpha}} \leq C\left(\|f\|_{L^{\infty}}+\|w\|_{L^{\infty}}\right) .
$$

(c2) If $2 \gamma>1$, then $f \in \mathcal{C}^{1, \alpha}\left(\mathbb{H}^{n}\right)$ for any $\alpha<2 \gamma-1$. Moreover,

$$
\|f\|_{\mathcal{C}^{1, \alpha}} \leq C\left(\|f\|_{L^{\infty}}+\|w\|_{L^{\infty}}\right) .
$$




\subsection{The extension problem}

For the rest of the section, we set $\gamma \in(0,1)$ and $a=1-2 \gamma$. We use the ideas in [11] to reduce the extension problem (1.1) to an ODE by taking Fourier transform in $x$. Let $u: \mathbb{H}^{n} \rightarrow \mathbb{R}_{+}$be a solution to

$$
\left\{\begin{aligned}
\partial_{y y} u+\frac{a}{y} \partial_{y} u+\Delta_{\mathbb{H}^{n}} u(x, y) & =0 & & \text { for }(x, y) \in \mathbb{H}^{n} \times \mathbb{R}_{+}, \\
u(x, 0) & =f(x) & & \text { for } x \in \mathbb{H}^{n},
\end{aligned}\right.
$$

where $g$ is the product metric on $\mathbb{H}^{n} \times \mathbb{R}_{+}$given by $g=g_{\mathbb{H} n}+d y^{2}$. Taking Fourier transform in the variable $x \in \mathbb{H}^{n}$, one obtains

$$
\left\{\begin{aligned}
\partial_{y y} \hat{u}+\frac{a}{y} \partial_{y} \hat{u}-\left(\lambda^{2}+\frac{(n-1)^{2}}{4}\right) \hat{u} & =0, \\
\hat{u}(\lambda, \theta, 0) & =\hat{f}(\lambda, \theta),
\end{aligned}\right.
$$

that is an ODE for each fixed value of $\lambda, \theta$. With the change of variables

$$
s=\left(\lambda^{2}+\frac{(n-1)^{2}}{4}\right)^{1 / 2} y, \quad \varphi(s)=\hat{u}(\cdot, y),
$$

it gets transformed to

$$
\partial_{s s} \varphi+\frac{a}{s} \partial_{s} \varphi-\varphi=0
$$

that is described in Lemma 2.3. Let $\varphi_{\gamma}$ the unique solution of (2.13) such that $\varphi_{\gamma}(0)=1, \varphi_{\gamma}(\infty)=0$, which is explicitly written as $\varphi_{\gamma}(s)=2^{1-\gamma} \Gamma(\gamma)^{-1} s^{\gamma} K_{\gamma}(s)$. Then the solution to (2.11) is simply the inverse Fourier transform on $\mathbb{H}^{n}$ of

$$
\hat{u}(\lambda, \theta, y)=\hat{f}(\lambda, \theta) \varphi_{\gamma}\left(\left(\lambda^{2}+\frac{(n-1)^{2}}{4}\right)^{1 / 2} y\right) .
$$

From the asymptotics at the origin, one may calculate

$$
\lim _{s \rightarrow 0} s^{a} \varphi_{\gamma}^{\prime}(s)=-d_{\gamma}^{-1}
$$

for the constant given in (1.3), and we have that

$$
\lim _{y \rightarrow 0} y^{a} \partial_{y} \hat{u}=\left(\lambda^{2}+\frac{(n-1)^{2}}{4}\right)^{(1-a) / 2} \hat{f} \lim _{s \rightarrow 0} s^{a} \varphi_{\gamma}^{\prime}(s)=-\left(\lambda^{2}+\frac{(n-1)^{2}}{4}\right)^{\gamma} \hat{f} d_{\gamma}^{-1}
$$

Taking the inverse Fourier transform in (2.16), we complete the proof of Theorem 1.1 in the case of hyperbolic space.

As we have mentioned, one of the advantages of the extension formulation (2.11) over the singular integral formulation from Theorem 2.5 is that it allows to prove elliptic type results. For instance, we show a weighted Hopf's maximum principle, which in the Euclidean case was considered in [12]. We note here that the proof only depends on the structure of equation (2.11). 
Proposition 2.7. Let $p \in \mathbb{H}^{n}$ set $B_{R}(p)$ to be the ball in $\mathbb{H}^{n}$ centered at $p$ of radius $R$. Consider the cylinder $C_{R, 1}=B_{R}(p) \times(0,1) \subset \mathbb{H}^{n} \times \mathbb{R}_{+}$. Let $u \in$ $\left(\overline{C_{R, 1}}\right) \cap H^{1}\left(C_{R, 1}, y^{a}\right)$ satisfy

$$
\left\{\begin{aligned}
\partial_{y y} u+\frac{a}{y} \partial_{y} u+\Delta_{\mathbb{H}^{n}} u & \leq 0 \quad \text { in } C_{R, 1}, \\
u & >0 \\
u(p, 0) & =0 .
\end{aligned}\right.
$$

Then $-\limsup _{y \rightarrow 0} y^{a} \frac{u(p, y)}{y}<0$. If, in addition, $y^{a} \partial_{y} u \in\left(\overline{C_{R, 1}}\right)$, then

$$
-\lim _{y \rightarrow 0} y^{a} \partial_{y} u(p, y)<0
$$

2.3.1. The Poisson kernel. In view of (2.14), we obtain that the solution of $(1.1)$ is

$$
\begin{aligned}
u(x, y) & =\int_{\mathbb{H}^{n}} f\left(x^{\prime}\right) \int_{-\infty}^{\infty} k_{\lambda}\left(x, x^{\prime}\right) \varphi_{\gamma}\left(\left(\lambda^{2}+\frac{(n-1)^{2}}{4}\right)^{1 / 2} y\right) d \lambda d x^{\prime} \\
& =: \int_{\mathbb{H}^{n}} f\left(x^{\prime}\right) \mathcal{P}_{y}^{\gamma}\left(d_{\mathbb{H}^{n}}\left(x, x^{\prime}\right)\right) d x^{\prime} .
\end{aligned}
$$

Therefore, from formula (2.2) the Poisson kernel may be written as

$$
\mathcal{P}_{y}^{\gamma}(\rho)=\int_{-\infty}^{\infty} k_{\lambda}(\rho) \varphi_{\gamma}\left(\left(\lambda^{2}+\frac{(n-1)^{2}}{4}\right)^{1 / 2} y\right) d \lambda
$$

where $k_{\lambda}(\rho)$ is defined in (2.5) and (2.6). For instance, for $n=3$, dimension for which the computations should be the simplest, the Poisson kernel is

$$
\mathcal{P}_{y}^{\gamma}(\rho)=\frac{1}{\sinh \rho} \int_{-\infty}^{\infty} \varphi_{\gamma}\left(\left(\lambda^{2}+\frac{(n-1)^{2}}{4}\right)^{1 / 2} y\right) \lambda \sin \lambda \rho \lambda d \lambda .
$$

We may now conclude the proof of Theorem 2.5. One has, by using successively (2.16), (2.17), and (2.11), and the fact that the Poisson kernel has integral equal to one (this is due to the fact that the extension solution for the constant function $f \equiv 1$ is the constant function $u \equiv 1$ ):

$$
\begin{aligned}
d_{\gamma}^{-1}\left(-\Delta_{\mathbb{H}^{n}}\right)^{\gamma} f & =-\lim _{y \rightarrow 0} y^{a} \partial_{y} u=-\lim _{y \rightarrow 0} y^{a} \frac{u(x, y)-u(x, 0)}{y} \\
& =-\int_{\mathbb{H}^{n}} \lim _{y \rightarrow 0} y^{a} \frac{\mathcal{P}_{y}^{\gamma}\left(d_{\mathbb{H}^{n}}\left(x, x^{\prime}\right)\right)}{y}\left(f\left(x^{\prime}\right)-f(x)\right) d x^{\prime} \\
& =-\int_{\mathbb{H}^{n}} \lim _{y \rightarrow 0} y^{a} \frac{\mathcal{P}_{y}^{\gamma}\left(d_{\mathbb{H}^{n}}\left(x, x^{\prime}\right)\right)-\mathcal{P}_{0}^{\gamma}\left(d_{\mathbb{H}^{n}}\left(x, x^{\prime}\right)\right)}{y}\left(f\left(x^{\prime}\right)-f(x)\right) d x^{\prime} \\
& =-\int_{\mathbb{H}^{n}} \lim _{y \rightarrow 0} y^{a} \partial_{y} \mathcal{P}_{y}^{\gamma}\left(d_{\mathbb{H}^{n}}\left(x, x^{\prime}\right)\right)\left(f\left(x^{\prime}\right)-f(x)\right) d x^{\prime} \\
& =-\int_{\mathbb{H}^{n}} \lim _{y \rightarrow 0} a_{\gamma}\left(y, d_{\mathbb{H}^{n}}\left(x, x^{\prime}\right)\right)\left(f\left(x^{\prime}\right)-f(x)\right) d x^{\prime},
\end{aligned}
$$


with

$$
\begin{aligned}
a_{\gamma}(y, \rho) & =\int_{-\infty}^{\infty} k_{\lambda}(\rho)\left(\lambda^{2}+\frac{(n-1)^{2}}{4}\right)^{1 / 2} y^{a} \varphi_{\gamma}^{\prime}\left(\left(\lambda^{2}+\frac{(n-1)^{2}}{4}\right)^{1 / 2} y\right) d \lambda \\
& =\int_{-\infty}^{\infty} k_{\lambda}(\rho)\left(\lambda^{2}+\frac{(n-1)^{2}}{4}\right)^{\gamma} s^{a} \varphi_{\gamma}^{\prime}(s) d \lambda,
\end{aligned}
$$

for $s=\left(\lambda^{2}+(n-1)^{2} / 4\right)^{1 / 2} y$. In view of the limit (2.15) we have

$$
\lim _{y \rightarrow 0} a_{\gamma}(y, \rho)=-d_{\gamma}^{-1} \mathcal{K}_{\gamma}(\rho)
$$

Now, since from Theorem 2.4 the kernel $\mathcal{K}_{\gamma}(\rho)$ behaves near zero as $\rho^{-n-2 \gamma}$ and decays exponentially at infinity, in the last integral in (2.18) we cannot replace the $\operatorname{limit}_{\lim _{y \rightarrow 0}} a_{\gamma}(y, \rho)$ by $-d_{\gamma}^{-1} \mathcal{K}_{\gamma}(\rho)$ because of the singular behavior at $\rho=0$. In order to make sense of the integration near zero the principal value has to be introduced, as it is done also in the Euclidean case (see for instance [11]). We obtain then

$$
\left(-\Delta_{\mathbb{H}^{n}}\right)^{\gamma} f=P . V . \int_{\mathbb{H}^{n}}\left(f\left(x^{\prime}\right)-f(x)\right) \mathcal{K}_{\gamma}\left(d_{\mathbb{H}^{n}}\left(x, x^{\prime}\right)\right) d x^{\prime},
$$

where now the right hand side is well defined. Indeed, by doing a Taylor development for $x^{\prime}$ near $x$ and using local charts, the first order term vanishes by parity, and the second order term behaves like $\rho^{\alpha-n-2 \gamma}$ if $f \in \mathcal{C}^{\alpha}$, which is integrable at zero if $\alpha>2 \gamma$. This completes the proof of Theorem 2.5.

Remark 2.8. The definition of the fractional Laplacian coming from the extension problem (2.11) also allows us to show that $\left(-\Delta_{\mathbb{H}^{n}}\right)^{\gamma}$ is a positive operator. This is so because the first eigenvalue may be computed using this formulation. If $U_{f}(\cdot, y)=\mathcal{P}_{y}^{\gamma} * f$,

$$
\begin{aligned}
\lambda_{1} & =\inf _{f} \frac{\int_{\mathbb{H}^{n}} f\left(-\Delta_{\mathbb{H}^{n}}\right)^{\gamma} f}{\int_{\mathbb{H}^{n}} f^{2}}=\inf _{f} \frac{-\int_{\mathbb{H}^{n}} f d_{\gamma} \lim _{y \rightarrow 0}\left(y^{a} \partial_{y} U_{f}(\cdot, y)\right)}{\int_{\mathbb{H}^{n}} f^{2}} \\
& =\inf _{U} \frac{\int_{\mathbb{H}^{n} \times \mathbb{R}_{+}} y^{a}\left|\nabla_{\mathbb{H}^{n}} U\right|^{2}}{\int_{\mathbb{H}^{n}}\left(\left.U\right|_{y=0}\right)^{2}} \geq 0 .
\end{aligned}
$$

This shows that the linear operator $\left(-\Delta_{\mathbb{H}^{n}}\right)^{\gamma}$ is non-negative, so its kernel $\mathcal{K}_{\gamma}$ must be non-negative too.

2.3.2. Energy formulation. We would like to re-write the weighted Dirichlet energy

$$
\|\nabla u\|_{L^{2}\left(\mathbb{H}^{n} \times \mathbb{R}_{+}, y^{a}\right)}^{2}=\int_{\mathbb{H}^{n} \times \mathbb{R}_{+}} y^{a}\left|\nabla_{g} u\right|^{2} d x d y .
$$

From (2.14) we obtain

$$
\widehat{\partial_{y} u}(\lambda, \theta, y)=\hat{f}(\lambda, \theta)\left(\lambda^{2}+\frac{(n-1)^{2}}{4}\right)^{1 / 2} \varphi_{\gamma}^{\prime}\left(\left(\lambda^{2}+\frac{(n-1)^{2}}{4}\right)^{1 / 2} y\right) \text {. }
$$


Using Plancherel's formula,

$$
\begin{aligned}
\int_{\mathbb{H}^{n}}\left|\nabla_{\mathbb{H}^{n}} f(x)\right|^{2} d x & =\int_{\mathbb{H}^{n}} f(x)\left(-\Delta_{\mathbb{H}^{n}} \bar{f}(x)\right) d x \\
& =\int_{-\infty}^{\infty} \int_{\mathbb{S}^{n-1}} \hat{f}(\lambda, \theta)\left(\widehat{\left.\Delta_{\mathbb{H}^{n}}\right)} \bar{f}(\lambda, \theta) \frac{d \theta d \lambda}{|c(\lambda)|^{2}}\right. \\
& =\int_{-\infty}^{\infty} \int_{\mathbb{S}^{n-1}}|\hat{f}(\lambda, \theta)|^{2}\left(\lambda^{2}+\frac{(n-1)^{2}}{4}\right) \frac{d \theta d \lambda}{|c(\lambda)|^{2}}
\end{aligned}
$$

Substituting the previous expressions in (2.19) and using Plancherel's formula we infer that

$$
\begin{aligned}
&\|\nabla u\|_{L^{2}\left(\mathbb{H}^{n} \times \mathbb{R}_{+}, y^{a}\right)}^{2}=\int_{\mathbb{H}^{n} \times \mathbb{R}_{+}}\left(\left|\partial_{y} u\right|^{2}+\left|\nabla_{\mathbb{H}^{n}} u\right|^{2}\right) y^{a} d x d y \\
&= \int_{-\infty}^{\infty} \int_{\mathbb{S}^{n-1}} \int_{0}^{\infty}\left(\left|\varphi_{\gamma}\right|^{2}+\left|\varphi_{\gamma}^{\prime}\right|^{2}\right)\left(\left(\lambda^{2}+\frac{(n-1)^{2}}{4}\right)^{1 / 2} y\right) \\
& \cdot|\hat{f}(\lambda, \theta)|^{2}\left(\lambda^{2}+\frac{(n-1)^{2}}{4}\right) y^{a} d y \frac{d \theta d \lambda}{|c(\lambda)|^{2}}
\end{aligned}
$$

By performing again the change of variables (2.12), we obtain

$$
\begin{aligned}
& \|\nabla u\|_{L^{2}\left(\mathbb{H}^{n} \times \mathbb{R}_{+}, y^{a}\right)}^{2} \\
& =\int_{-\infty}^{\infty} \int_{\mathbb{S}^{n-1}}|\hat{f}(\lambda, \theta)|^{2}\left(\lambda^{2}+\frac{(n-1)^{2}}{4}\right)^{\gamma} \int_{0}^{\infty}\left(\left|\varphi_{\gamma}(s)\right|^{2}+\left|\varphi_{\gamma}^{\prime}(s)\right|^{2}\right) \mid s^{a} d s \frac{d \theta d \lambda}{|c(\lambda)|^{2}} \\
& =C_{\gamma} \int_{\mathbb{H}^{n}}\left|\left(-\Delta_{\mathbb{H}^{n}}\right)^{\gamma} f(x)\right|^{2} d x,
\end{aligned}
$$

where

$$
C_{\gamma}=I\left[\varphi_{\gamma}\right]:=\int_{0}^{\infty}\left(\left|\varphi_{\gamma}(s)\right|^{2}+\left|\varphi_{\gamma}^{\prime}(s)\right|^{2}\right) s^{a} d s
$$

is a positive constant that only depends on $\gamma$. Note that $\varphi_{\gamma}$ is the minimizer of the functional $I[\varphi]$. In order to calculate the precise value of this constant, we multiply equation (2.13) by $s^{\gamma} \varphi_{\gamma}$ and integrate between $\epsilon$ and $\infty$. Then

$$
C_{\gamma}=\lim _{\epsilon \rightarrow 0} \int_{\epsilon}^{\infty}\left(\left|\varphi_{\gamma}(s)\right|^{2}+\left|\varphi_{\gamma}^{\prime}(s)\right|^{2}\right) s^{a} d s=-\lim _{\epsilon \rightarrow 0} \epsilon^{a} \varphi_{\gamma}(\epsilon) \varphi_{\gamma}^{\prime}(\epsilon)=d_{\gamma}^{-1},
$$

according to $(2.15)$.

After all this discussion, one may show, as in the real case ([11], [18]), that:

Theorem 2.9 (Trace Sobolev embedding). For every $u \in W^{1,2}\left(\mathbb{H}^{n} \times \mathbb{R}_{+}, y^{a}\right)$, we have that

$$
\|\nabla u\|_{L^{2}\left(\mathbb{H}^{n} \times \mathbb{R}_{+}, y^{a}\right)}^{2} \geq d_{\gamma}^{-1}\|u(\cdot, 0)\|_{\dot{H}^{\gamma}\left(\mathbb{H}^{n}\right)}^{2}
$$

for the constant given in (1.3), and with equality if and only if $u$ is the Poisson extension (2.17) of some function in $\dot{H}^{\gamma}\left(\mathbb{H}^{n}\right)$. 


\subsection{Other (noncompact) harmonic groups}

The calculations in this section for the real hyperbolic space rely on the harmonic analysis available in this setting, by following the arguments done in [11] for the Euclidean case. In the same spirit, one can start to perform the same arguments in the case of more general harmonic groups as Damek-Ricci spaces, also known as harmonic NA groups. All symmetric spaces of rank one are included in this class. Part of the importance of this class is that it also contains non symmetric spaces, thus providing counterexamples to the Lichnerowicz conjecture (see [19] an [3]; see also [5]).

Instead of pursuing this method, we take the point of view of the next section using the heat kernel. Moreover, in Remark 3.4 we show that in several examples of this family, the fractional Laplacian can be represented as singular integral, where precise asymptotics can be given for the kernel.

\section{The fractional Laplacian on noncompact manifolds}

The aim of this section is to construct the fractional Laplacian on a noncompact manifold $M$ through the extension problem (1.1), and to give sufficient conditions for the existence of a Poisson kernel. The main tool here is the study of the heat kernel on $M$; geometry plays a fundamental role.

\subsection{From heat to Poisson}

First we give some standard functional analysis background from [59], [69]. Let $L$ be a linear second order partial differential operator on $M$, that is assumed to be nonnegative, densely defined and self-adjoint in $L^{2}(M)$, for instance, $L=-\Delta_{M}$ for a complete manifold $M$. Then the spectral theorem can be applied to $L$, and consequently, there exists a unique resolution $E$ of the identity, supported on the spectrum of $L$ (which is a subset of $[0, \infty)$ ), such that

$$
L=\int_{0}^{\infty} \lambda d E(\lambda) .
$$

Given a real measurable function $h$ on $[0, \infty)$, the operator $h(L)$ is formally defined as $h(L)=\int_{0}^{\infty} h(\lambda) d E(\lambda)$. The domain $\operatorname{Dom}(h(L))$ of $h(L)$ is the set of functions $f \in L^{2}(\Omega)$ such that $\int_{0}^{\infty}|f(\lambda)|^{2} d E_{h, h}(\lambda)<\infty$. In particular, one may define the heat diffusion semigroup generated by $L$ as $h(L)=e^{-t L}, t \geq 0$. Then:

1. For $f \in L^{2}(M)$, we have that $u=e^{-t L} f$ solves the evolution equation $u_{t}=-L u$, for $t>0$.

2. $\left\|e^{-t L} f\right\|_{L^{2}(M)} \leq\|f\|_{L^{2}(M)}$, for all $t \geq 0$.

3. $e^{-t L} f \rightarrow f$ in $L^{2}(M)$ as $t \rightarrow 0^{+}$.

One may also consider the fractional powers of $L$, given by $h(L)=L^{\gamma}, \gamma \in(0,1)$, with domain $\operatorname{Dom}\left(L^{\gamma}\right) \supset \operatorname{Dom}(L)$. Then: 
1. When $f \in \operatorname{Dom}\left(L^{\gamma}\right)$, we have $L^{\gamma} e^{-t L} f=e^{-t L} L^{\gamma} f$.

2. If $f \in \operatorname{Dom}(L)$, then $\langle L f, f\rangle=\left\|L^{1 / 2} f\right\|_{L^{2}(M)}^{2}$, where $\langle\cdot, \cdot\rangle$ denotes the inner product in $L^{2}(M)$.

3. For $f \in \operatorname{Dom}(L)$,

$$
L^{\gamma} f(x)=\frac{1}{\Gamma(-\gamma)} \int_{0}^{\infty}\left(e^{-t L} f(x)-f(x)\right) \frac{d t}{t^{1+\gamma}}, \quad \text { in } L^{2}(M) .
$$

In this framework, the paper [62] relates the heat semigroup to the extension problem (1.1) for the fractional Laplacian (their work is for domains in $\mathbb{R}^{n}$ with a measure, but it is easily generalized when $M$ is a $n$-dimensional manifold). Let $f \in D\left(L^{\gamma}\right)$, and consider the extension problem

$$
\left\{\begin{array}{rlrl}
\partial_{y y} u+\frac{a}{y} \partial_{y} u-L_{x} u & =0 & & \text { in } \\
u(\cdot, 0) & =f \quad & \text { on } \quad M .
\end{array}\right.
$$

They show that:

Theorem 3.1. A solution to (3.1) is given by

$$
u(x, y)=\frac{1}{\Gamma(\gamma)} \int_{0}^{\infty} e^{-t L}\left(L^{\gamma} f\right)(x) e^{-\frac{y^{2}}{4 t}} \frac{d t}{t^{1-\gamma}}
$$

and

$$
\lim _{y \rightarrow 0^{+}} \frac{u(x, y)-u(x, 0)}{y^{2 \gamma}}=\frac{1}{2 \gamma} \lim _{y \rightarrow 0^{+}} y^{a} \partial_{y} u(x, y)=\frac{\Gamma(-\gamma)}{2^{2 \gamma} \Gamma(\gamma)} L^{\gamma} f(x) .
$$

Moreover, the following Poisson formula for $u$ holds:

$$
\begin{aligned}
u(x, y) & =\frac{y^{2 \gamma}}{2^{2 \gamma} \Gamma(\gamma)} \int_{0}^{\infty} e^{-t L} f(x) e^{-\frac{y^{2}}{4 t}} \frac{d t}{t^{1+\gamma}} \\
& =\frac{1}{\Gamma(\gamma)} \int_{0}^{\infty} e^{-\frac{y^{2}}{4 r} L} f(x) e^{-r} \frac{d r}{r^{1-\gamma}}=: P_{y}^{\gamma} f(x)
\end{aligned}
$$

These identities are to be understood in the $L^{2}$ sense. If addition, we make the following extra assumptions:

(I) The heat diffusion semigroup is given by integration against a nonnegative heat kernel $p_{t}(x, \zeta)$.

(II) The heat kernel belongs to the domain of $L$ and $\partial_{t} p_{t}=L p_{t}$, where the $t$-derivative is understood in the classical sense.

(III) Given $x$, there exists a constant $C_{x}$ and $\epsilon>0$ such that

$$
\left\|p_{t}(x, \cdot)\right\|_{L^{2}(M)}+\left\|\partial_{t} p_{t}(x, \cdot)\right\|_{L^{2}(M)} \leq C_{x}\left(1+t^{\epsilon}\right) t^{-\epsilon} .
$$

In the same paper [62], the authors give a formula for the Poisson kernel for (3.1). 
Theorem 3.2. Under the additional hypotheses (I), (II) and (III) we have:

(1) One may write $P_{y}^{\gamma} f(x)=\int_{M} \mathcal{P}_{y}^{\gamma}(x, \zeta) f(\zeta) d v(\zeta)$, where the Poisson kernel is given by

$$
\mathcal{P}_{y}^{\gamma}(x, \zeta):=\frac{y^{2 \gamma}}{2^{2 \gamma} \Gamma(\gamma)} \int_{0}^{\infty} p_{t}(x, \zeta) e^{-\frac{y^{2}}{4 t}} \frac{d t}{t^{1+\gamma}},
$$

and, for each $\zeta \in M$, is a $L^{2}$-function that verifies the first equation in (3.2).

(2) $\sup _{y \geq 0}\left|P_{y}^{\gamma} f\right| \leq \sup _{t \geq 0}\left|e^{-t L} f\right|$ in $M$.

(3) $\left\|P_{y}^{\gamma} f\right\|_{L^{p}(M)} \leq\|f\|_{L^{p}(M)}$, for all $y \geq 0$.

(4) If $\lim _{t \rightarrow 0^{+}} e^{-t L} f=f$ in $L^{p}(M)$, then $\lim _{y \rightarrow 0^{+}} P_{y}^{\gamma} f=f$ in $L^{p}(M)$.

\subsection{Heat kernels on noncompact manifolds}

Good references for heat kernel on manifolds are the book [20] or the survey [38]. Let $\left(M^{n}, g\right)$ be a Riemannian manifold with a metric $g$. Let $L=-\Delta_{M}$, the Laplace-Beltrami operator with respect to this metric. If $M$ is complete, then $L$ is self-adjoint on $\mathcal{C}_{0}^{\infty}(M)$. We have that $e^{-L t}$ is a positivity-preserving one-parameter contraction semigroup on $L^{p}(M)$ for $1 \leq p \leq \infty$. In particular, in $L^{2}(M)$ it has a strictly positive $\mathcal{C}^{\infty}$ kernel $p_{t}(x, y), x, x^{\prime} \in M, t>0$, satisfying

(a) as a function of $t$ and $x$,

$$
\partial_{t} p_{t}=\Delta_{M} p_{t}
$$

(b) when $t \rightarrow 0^{+}$,

$$
p_{t}\left(\cdot, x^{\prime}\right) \rightarrow \delta_{x^{\prime}}
$$

(c) the semigroup property $e^{(t+s) \Delta_{M}}=e^{t \Delta_{M}} e^{s \Delta_{M}}$, which reads as

$$
p_{t+s}\left(x, x^{\prime}\right)=\int_{M} p_{t}(x, z) p_{s}\left(x, x^{\prime}\right) d z
$$

(d) and the symmetry

$$
p_{t}\left(x, x^{\prime}\right)=p_{t}\left(x^{\prime}, x\right) .
$$

It is clear then that the hypothesis of Theorem 3.1 are satisfied in this case. However, to pass from the heat kernel to the Poisson kernel as in Theorem 3.2 is a non-trivial issue in the case of non-compact manifolds since one needs to control the behavior at infinity.

We concentrate here into obtaining the bound (III). First, in order to get $L^{2}$ estimates for the kernel $p_{t}$ it is enough to have $L^{\infty}$ bounds. Indeed, for fixed $x \in M$,

$$
\left\|p_{t}(x, \cdot)\right\|_{L^{2}(M)}^{2} \leq \sup _{x^{\prime} \in M}\left\{p_{t}\left(x, x^{\prime}\right)\right\} \int_{M} p_{t}\left(x, x^{\prime}\right) d x^{\prime} \leq\left\|p_{t}(x, \cdot)\right\|_{L^{\infty}(M)},
$$

where we have used that, for all $t>0$ and $x \in M, \int_{M} p_{t}\left(x, x^{\prime}\right) d x^{\prime} \leq 1$, where $d x^{\prime}$ is the volume element in the manifold $M$. For stochastically complete manifolds, this integral is exactly equal to one (see [36]). 
Now, to estimate the time derivative of $p_{t}$, we recall that (see [37])

$$
\int_{M}\left|\nabla^{k} p_{t}\left(x, x^{\prime}\right)\right|^{2} d x^{\prime} \leq \frac{1}{k} \int_{M}\left|p_{t / 2^{k}}\left(x, x^{\prime}\right)\right|^{2}, \quad \text { for all } k \in \mathbb{N} .
$$

We conclude that, in order to check hypothesis (III) in Theorem 3.2, it is enough to find good pointwise upper bounds for the heat kernel $p_{t}\left(x, x^{\prime}\right)$. This can be also seen by the holomorphy of the heat semigroup (see for instance [68]).

On the other hand, because of the semigroup property (3.3) and the symmetry of the heat kernel,

$$
p_{t}(x, x)=\int_{M} p_{t / 2}(x, z)^{2} d z .
$$

Using the semigroup identity (3.3) again and the Cauchy-Schwarz inequality,

$p_{t}\left(x, x^{\prime}\right)=\int_{M} p_{t / 2}(x, z) p_{t / 2}\left(x^{\prime}, z\right) d z \leq\left(\int_{M} p_{t / 2}(x, z)^{2} d z\right)^{1 / 2}\left(\int_{M} p_{t / 2}\left(x^{\prime}, z\right)^{2} d z\right)^{1 / 2}$,

which together with (3.4) implies

$$
p_{t}\left(x, x^{\prime}\right) \leq \sqrt{p_{t}(x, x) p_{t}\left(x^{\prime}, x^{\prime}\right)} ;
$$

i.e., if one knew good on-diagonal estimates for $p_{t}(x, x)$, this would imply a $L^{\infty}$ bound for the heat kernel $p_{t}\left(x, x^{\prime}\right)$. Also, we get $p_{2 t}(x, x) \leq p_{t}(x, x)$ so if (III) is satisfied up to some time $t_{0}$, then we get for all $t \geq t_{0}$ the upper bound $C_{x}(1+$ $\left.\left(t_{0} / 2\right)^{\epsilon}\right)\left(t_{0} / 2\right)^{-\epsilon}$. Therefore it is enough to verify (III) for small times.

Note that the heat kernel on Euclidean space has the explicit formula

$$
p_{t}\left(x, x^{\prime}\right)=\frac{1}{(4 \pi t)^{n / 2}} e^{-\frac{\rho^{2}}{4 t}}
$$

where $\rho=\left|x-x^{\prime}\right|$. The survey [38] gives many examples for bounds of the heat kernel, in relation to Faber-Krahn and isoperimetric inequalities. For instance,

- minimal submanifolds of $\mathbb{R}^{N}$;

- manifolds with sectional curvature bounded in between two constants ([16]);

- Cartan-Hadamard manifolds; which are geodesically complete simply connected non-compact Riemannian manifolds with non-positive sectional curvature $([14],[24])$.

In all of these the heat kernel has the same asymptotic behavior as in the Euclidean case:

$$
0 \leq p_{t}\left(x, x^{\prime}\right) \leq \frac{C}{t^{n / 2}} e^{-\frac{\rho^{2}}{c t}}
$$

for some $c, C>0$, and where $\rho=d_{M}\left(x, x^{\prime}\right)$.

If $M$ is a complete manifold with non-negative Ricci tensor, heat kernel bounds were obtained by [49]. More generally, if $M$ is a complete manifold with lower bounded Ricci tensor,

$$
\operatorname{Ric} \geq-(n-1) \beta^{2}
$$


for some $\beta>0$, then, denoting $\lambda_{1} \geq 0$ the bottom of the spectrum of the operator $-\Delta_{M}$, we have the following heat kernel estimate [21]:

$$
0 \leq p_{t}\left(x, x^{\prime}\right) \leq c_{\delta}|B(x, \sqrt{t})|^{-1 / 2}\left|B\left(x^{\prime}, \sqrt{t}\right)\right|^{-1 / 2} e^{\left(\delta-\lambda_{1}\right) t} e^{-\frac{\rho^{2}}{(4+\delta) t}} .
$$

We recall Bishop's comparison theorem, which states that if $0<R_{1}<R_{2}$, then

$$
\frac{\left|B\left(x, R_{2}\right)\right|}{\left|B\left(x, R_{1}\right)\right|} \leq \frac{\left|B_{\beta}\left(R_{2}\right)\right|}{\left|B_{\beta}\left(R_{1}\right)\right|},
$$

where $B_{\beta}(R)$ denotes the volume of the geodesic ball of radius $R$ in the constant $-\beta^{2}$ sectional curvature space form, that may be calculated from the volume element

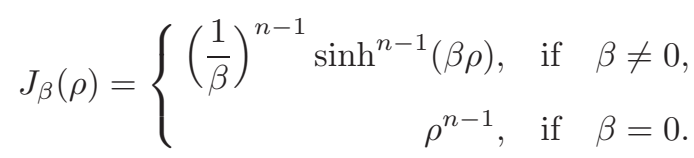

In particular, for all $x \in M$,

$$
\frac{1}{|B(x, \sqrt{t})|} \leq \frac{1}{|B(x, 1)|} \frac{C}{t^{n}}
$$

It is clear that to prove the bound (III) in the situations just discussed, one should ask for a lower bound for the volume of balls. More precisely, we seek $C>0$ such that

$$
|B(x, 1)| \geq C>0 \quad \forall x \in M .
$$

Finally, for a manifold with doubling condition and local Poincaré inequality, we have as an upper-bound for the heat kernel [60]

$$
p_{t}(x, x) \leq \frac{C}{|B(x, \sqrt{t})|} .
$$

In view of the doubling condition, it is enough again to have (3.7) to get (III).

Summarizing the results of this section:

Proposition 3.3. Let $M$ be a complete noncompact Riemannian manifold. Hypothesis (III) in Theorem 3.2 is fulfilled if $M$ satisfies any of these:

- $M$ is a Cartan-Hadamard manifold.

- $\operatorname{Ric} \geq-(n-1) \beta^{2}$ for some $\beta>0$ and (3.7) holds.

- $M$ satisfies a volume doubling condition and the local Poincaré inequality.

In the following, we look at several classes of admissible manifolds. The first two examples are classical and more or less explicit formulas for the heat kernel are known. Then we concentrate on spherically symmetric manifolds, that serve as a model for more general cases such as manifolds with ends. 


\subsection{Admissible classes of manifolds}

3.3.1. Symmetric spaces. An explicit expression can be found for the heat kernel on hyperbolic space:

$$
p_{t}(\rho)=\frac{(2 \pi)^{-d / 2}}{2} t^{-1 / 2} e^{-\left(\frac{n-1}{2}\right)^{2} t}\left(-\frac{\partial_{\rho}}{\sinh \rho}\right)^{(n-1) / 2} e^{-\frac{\rho^{2}}{4 t}}
$$

for $n \geq 3$ odd, and for $n \geq 2$ even,

$$
p_{t}(\rho)=(2 \pi)^{-(d+1) / 2} t^{-1 / 2} e^{-\left(\frac{n-1}{2}\right)^{2} t} \int_{\rho}^{\infty} \frac{\sinh s}{\sqrt{\cosh s-\cosh \rho}}\left(\frac{\partial_{s}}{\sinh s}\right)^{n / 2} e^{-\frac{s^{2}}{4 t}} d s,
$$

where $\rho=d_{\mathbb{H}^{n}}\left(x, x^{\prime}\right)$. Moreover, global bounds were derived in [22]:

$$
p_{t}(\rho) \approx \frac{(1+\rho)(1+\rho+t)^{(n-3) / 2}}{t^{n / 2}} e^{-\frac{(n-1)^{2}}{4} t-\frac{n-1}{2} \rho-\frac{\rho^{2}}{4 t}},
$$

for all $\rho \geq 0, t>0$, in the sense that the ratio of the right-hand side and the lefthand side is uniformly bounded between two positive constants. These estimates have a generalisation to all symmetric spaces of rank one, and more generally to Damek-Ricci spaces, since in this case there is also an explicit formula for the heat kernel.

Global bounds on the heat kernel in the higher rank case were proved in [6]. We recall that Ricci curvature is bounded from below since is an Einstein manifold, that is Ric $=-\left(m_{2} / 4+m_{1}\right) g$ (where as before $g$ is the Riemannian metric on $M$ ). Here $m_{1}$ and $m_{2}$ stand for the two dimensions entering the algebraic construction of the space. For instance $m_{1}=0, m_{2}=n-1$ for the real hyperbolic space and $m_{1}=1, m_{2}=2(n-1)$ for the complex hyperbolic space. Also, the volume element is of type $\sinh r^{m_{1}+m_{2}} \cosh r^{m_{1}}$. Therefore Theorem 3.2 applies by using directly the explicit sharp $L^{\infty}$ bounds on the heat kernel, or by using (3.7) and explicit formulas for the volume of the balls. Moreover, we can represent the fractional Laplacian in the spirit of Theorem 2.5.

Remark 3.4. From $[6]$ we have that

$\mathcal{P}_{y}^{\gamma}(x, \zeta) \approx \int_{0}^{\infty} \frac{\prod_{\alpha \in \Sigma_{0}^{+}}(1+\langle\alpha, H\rangle)(1+\langle\alpha, H\rangle+t)^{\frac{m_{\alpha}+m_{2 \alpha}}{2}-1}}{t^{n / 2+1+\gamma}} e^{-|\varrho|^{2} t-\langle\varrho, H\rangle-\frac{|H|^{2}}{4 t}} d t$.

The quantities $\varrho, H, \alpha$ and $\Sigma_{0}$ are related to the Cartan decomposition as follows: if the associated Lie algebra $\mathfrak{g}$ decomposes as

$$
\mathfrak{g}=\mathfrak{a} \oplus \mathfrak{m} \oplus\left\{\oplus_{\alpha \in \Sigma} \mathfrak{g}_{\alpha}\right\},
$$

and $\Sigma^{+}, \Sigma_{0}^{+}$denote the set of positive and positive indivisible roots, then

$$
\begin{aligned}
\varrho & =\frac{1}{2} \sum_{\alpha \in \Sigma^{+}} m_{\alpha} \alpha, \text { where } m_{\alpha}=\operatorname{dim} \mathfrak{g}_{\alpha}, \quad G=K\left(\exp \overline{\mathfrak{a}^{+}}\right) K, \\
\zeta^{-1} x & =k_{1} e^{H} k_{2}, \quad \text { with } k_{1}, k_{2} \in K, H \in \overline{\mathfrak{a}^{+}} .
\end{aligned}
$$

For precise definitions of these quantities we refer the reader to [6] and [46]. 
In a similar fashion as the proof of Theorem 2.5, one can find an explicit formula for the convolution kernel of the fractional Laplacian. From Theorem 3.2 we have that obtaining this kernel is equivalent to compute the Laplace transform of the function

$$
\prod_{\alpha \in \Sigma_{0}^{+}}(1+\langle\alpha, H\rangle)(1+\langle\alpha, H\rangle+t) \frac{\frac{m_{\alpha}+m_{2 \alpha}}{2}}{2} \frac{e^{-\langle\varrho, H\rangle-\frac{|H|^{2}}{4 t}}}{t^{n / 2+1+\gamma}} \text { at }|\varrho|^{2} .
$$

The Laplace transform of

$$
g(t)=\frac{e^{-\langle\varrho, H\rangle-\frac{|H|^{2}}{4 t}}}{t^{n / 2+1+\gamma}}
$$

is given by the integral

$$
\mathcal{L}(g)(s)=\int_{0}^{\infty} g(t) e^{-s t} d t
$$

Since

$$
t^{2} g^{\prime}(t)=\left(\frac{|H|^{2}}{4}-\left(\frac{n}{2}+1+\gamma\right) t\right) g(t),
$$

and $g(0)=0$, applying Laplace transform we have

$$
\partial_{s}^{2}(s \mathcal{L}(g))=\left(\frac{|H|^{2}}{4}+\left(\frac{n}{2}+1+\gamma\right) \partial_{s}\right) \mathcal{L}(g),
$$

which is a Bessel type equation of solution

$$
\mathcal{L}(g)=C_{n, \gamma} s^{(n+2 \gamma) / 4} K_{(n+2 \gamma) / 2}\left(|H| s^{1 / 2}\right),
$$

with $K_{\nu}$ given by Lemma 2.3 .

On the other hand, a simple computation shows that the Laplace transform of $(1+\langle\alpha, H\rangle+t) \frac{m_{\alpha}+m_{2 \alpha}}{2}-1$ is given by

$$
\frac{e^{s+1+\langle\alpha, H\rangle}}{s^{\frac{m_{\alpha}+m_{2 \alpha}}{2}-1}} \Gamma\left(\frac{m_{\alpha}+m_{2 \alpha}}{2}\right) .
$$

Since

$$
\mathcal{L}(g h)(s)=\frac{1}{2 \pi i} \lim _{T \rightarrow \infty} \int_{c-i T}^{c+i T} \mathcal{L}(g)(\sigma) \mathcal{L}(h)(\sigma-s) d \sigma,
$$

where the integration is done along the vertical line $\operatorname{Re}(z)=c$. We denote this convolution type of operation as $\mathcal{L}(g) \tilde{\star} \mathcal{L}(h)$.

Then, by denoting as $q_{i}=\left(m_{\alpha_{i}}+m_{2 \alpha_{i}}\right) / 2$ and $l$ the total number of roots in $\Sigma_{0}^{+}$, we can compute the desired kernel as

$$
\begin{aligned}
\mathcal{K}_{\gamma}\left(e^{H}\right)= & \frac{e^{s+1+\left\langle\alpha_{1}, H\right\rangle}}{s^{q_{1}-1}} \Gamma\left(q_{1}\right) \tilde{\star} \cdots \tilde{\star} \frac{e^{s+1+\left\langle\alpha_{l}, H\right\rangle}}{s^{q_{l}-1}} \Gamma\left(q_{l}\right) \\
& \left.\tilde{\star} C_{n, \gamma} s^{(n+2 \gamma) / 4} K_{(n+2 \gamma) / 2}\left(|H| s^{1 / 2}\right)\right|_{s=|\varrho|^{2}}
\end{aligned}
$$


3.3.2. Geometrically finite hyperbolic manifolds. The following introduction is standard and we refer the reader to [9], [57]. Let $\Gamma$ be a discrete group of isometries of $\mathbb{H}^{n}$, that without loss of generality can be taken to be torsion-free. Then the quotient $M=\mathbb{H}^{n} / \Gamma$ is a smooth manifold which inherits a complete hyperbolic structure. If $x \in \mathbb{H}^{n}$, the set of accumulation points of the orbit $x \Gamma$ in $\overline{\mathbb{H}^{n}}$ is a closed subset $\Lambda(\Gamma) \subset \mathbb{S}^{n-1}$ called the limit set of $\Gamma$. Its complement $\Omega(\Gamma)=\mathbb{S}^{n-1} \backslash \Lambda(\Gamma)$ is called the domain of discontinuity and $\Gamma$ acts properly discontinuously in $\Omega(\Gamma)$.

We assume, in addition, that $\Gamma$ is geometrically finite, i.e., it admits a fundamental domain with finitely many sides, and we consider those groups for which $M$ has infinite volume. In the case that no parabolic subgroup involves irrational rotations, which is the setting of [55] for the study of the resolvent of the Laplacian $\Delta_{M}$ (see also [42] for more general admissible groups), it is easy to give a geometrical description. In fact, there exists a compact $K$ of $M$ such that $M \backslash K$ is covered by a finite number of charts isometric to either a regular neighborhood $\left(M_{0}, g_{0}\right)$, where

$$
M_{0}=\left\{\left(x_{1}, x_{2}\right) \in(0, \infty) \times \mathbb{R}^{n-1}: x_{1}^{2}+\left|x_{2}\right|^{2}<1\right\}, \quad g_{0}=\left(x_{1}\right)^{-2}\left(d x_{1}^{2}+d x_{2}^{2}\right),
$$

or a rank- $r$ cusp neighborhood $\left(M_{r}, g_{r}\right), 1 \leq r \leq n-1$, where

$$
\begin{aligned}
M_{r} & =\left\{\left(x_{1}, x_{2}, x_{3}\right) \in(0, \infty) \times \mathbb{R}^{n-1-r} \times T^{r}: x_{1}^{2}+\left|x_{2}\right|^{2}>1\right\}, \\
g_{r} & =\left(x_{1}\right)^{-2}\left(d x_{1}^{2}+d x_{2}^{2}+d x_{3}^{2}\right),
\end{aligned}
$$

for $r<n-1$, and

$$
M_{n-1}=\left\{\left(x_{1}, x_{3}\right) \in(0, \infty) \times T^{n}: x_{1}>1\right\}, \quad g_{n-1}=\left(x_{1}\right)^{-2}\left(d x_{1}^{2}+d x_{3}^{2}\right) .
$$

Here $\left(T^{k}, d x_{3}^{2}\right)$ is a $k$-dimensional flat torus.

When such $\Gamma$ has no parabolic elements, then both $\Gamma$ and the quotient $\mathbb{H}^{n} / \Gamma$ are called convex co-compact, and the quotient manifold $M$ has no cusp then.

We define $\delta(\Gamma)$, the exponent of convergence of the Poincaré series, by

$$
\delta(\Gamma)=\inf \left\{s>0: \sum_{h \in \Gamma} d_{\mathbb{H}}\left(x, h x^{\prime}\right)^{-s}<\infty\right\},
$$

where $x, x^{\prime} \in \mathbb{H}^{n}$. Note that it depends upon the group $\Gamma$ but not upon the choice of $x, x^{\prime}$. It is known that $0 \leq \delta(\Gamma) \leq n-1$. We also define $\mu_{\alpha}$, for $\alpha>0$, by

$$
\mu_{\alpha}(x)=\left\{\sum_{h \in \Gamma} e^{-\alpha d_{\mathbb{H}} n(x, h x)}\right\}^{1 / 2},
$$

noting that the sum is invariant under the action of $\Gamma$ on $x$, so that $\mu_{\alpha}$ can also be regarded as a function on $\mathbb{H}^{n} / \Gamma$. Although the series converges for all $\alpha>\delta(\Gamma)$, we shall often assume that $\alpha>n-1$, because $\mu_{\alpha}$ is both smaller and easier to estimate for larger $\alpha$. We note that the distance function $\tilde{\rho}$ on $\mathbb{H}^{n} / \Gamma$ is given by

$$
\tilde{\rho}\left(x, x^{\prime}\right)=\min _{h \in \Gamma} d_{\mathbb{H}^{n}}\left(h x, x^{\prime}\right) .
$$


We recall the following bounds for the heat kernel on $\mathbb{H}^{n} / \Gamma$ from $[22]$. For $t \in(0, \infty)$ and any $\epsilon>0$ :

(i) If $0 \leq \delta(\Gamma)<(n-1) / 2$, then

$$
0 \leq \tilde{p}_{t}\left(x, x^{\prime}\right) \leq c_{\epsilon} t^{-n / 2} e^{-\left((n-1)^{2} / 4-2 \epsilon\right) t} e^{-\frac{\tilde{\rho}^{2}}{4(1+\epsilon) t}} \mu_{\alpha}(x) \mu_{\alpha}\left(x^{\prime}\right) .
$$

(ii) If $(n-1) / 2 \leq \delta(\Gamma) \leq n-1$ and $\alpha<\delta(\Gamma)$,

$$
p_{t}\left(x, x^{\prime}\right) \leq c_{\epsilon} t^{-n / 2} e^{-([\delta(\Gamma)(n-1-\delta(\Gamma))]-2 \epsilon) t} e^{-\frac{\tilde{\rho}^{2}}{4(1+\epsilon) t}} \mu_{\alpha}(x) \mu_{\alpha}\left(x^{\prime}\right) .
$$

Moreover, if $\alpha>n-1$, then $\mu_{\alpha}(x) \rightarrow 1$ as $x$ approaches to an end, and $\mu_{\alpha}(x) \sim$ $e^{\frac{1}{2} \rho(x, z) r}$ as $x$ approaches a cusp of rank $r$, where $z$ is any point in $\mathbb{H}^{n}$. In particular, $\mu_{\alpha}(x)$ is bounded if $\alpha>n-1$ and the manifold has no cusps.

In order to obtain $L^{\infty}$ estimates for the heat kernel near a cusp of rank $r$, we make the following observation:

$$
e^{-\frac{\rho^{2}}{4(1+\epsilon) t}} e^{\frac{r}{2} \rho}=e^{-\left(\frac{\rho}{2 \sqrt{(1+\epsilon) t}}-\frac{r \sqrt{(1+\epsilon) t}}{2}\right)^{2}} e^{\frac{r^{2}(1+\epsilon)}{4} t} .
$$

We get good $L^{\infty}$ bounds for $p_{t}(x, \cdot)$ when:

(i) $0 \leq \delta(\Gamma)<(n-1) / 2, r<n-1$ and there is no maximal cusp. Or,

(ii) $\delta(\Gamma)=(n-1) / 2+\beta / 2$ for some $\beta \in[0, n-1)$ and $r<(n-1)^{2}-\beta^{2}$.

Alternatively, we can obtain good bounds for the heat kernel if the manifold has no cusp.

3.3.3. Rotationally symmetric manifolds. We consider a noncompact rotationally symmetric manifold with a pole at the origin, i.e. a manifold $M^{n}$ given by the metric

$$
g_{M}=d r^{2}+\phi^{2}(r) d \omega^{2}
$$

where $d \omega^{2}$ is the metric on $\mathbb{S}^{n-1}$, and $\phi$ is a $\mathcal{C}^{\infty}$ nonnegative function on $[0, \infty)$, strictly positive on $(0, \infty)$, such that $\phi^{(\text {even })}(0)=0$ and $\phi^{\prime}(0)=1$. These conditions on $\phi$ ensure us that the manifold is smooth (see $\$ 1.3 .4$ of [56]). The volume element is $\phi^{n-1}(r) d r d \omega$, and the Laplace-Beltrami operator on $M$ is

$$
\Delta_{M}=\partial_{r r}+(n-1) \frac{\phi^{\prime}(r)}{\phi(r)} \partial_{r}+\frac{1}{\phi^{2}(r)} \Delta_{\mathbb{S}^{n-1}} .
$$

For such manifold, the curvature of $M$ can be computed explicitly in terms of $\phi$ (see $\S 3.2 .3$ of $[56])$. Indeed, there exists an orthonormal frame $\left(F_{j}\right)_{j=1}^{n}$ on $(M, g)$, where $F_{n}$ corresponds to the radial coordinate, and $F_{1}, \ldots, F_{n-1}$ to the spherical coordinates, for which $F_{i} \wedge F_{j}$ diagonalize the curvature operator $\mathcal{R}$ :

$\mathcal{R}\left(F_{i} \wedge F_{n}\right)=-\frac{\phi^{\prime \prime}}{\phi} F_{i} \wedge F_{n}, i<n, \quad$ and $\quad \mathcal{R}\left(F_{i} \wedge F_{j}\right)=-\frac{\left(\phi^{\prime}\right)^{2}-1}{\phi^{2}} F_{i} \wedge F_{j}, i, j<n$, 
which gives the sectional curvature

$$
\begin{array}{ll}
K_{i i}=0, & i=1, \ldots, n, \\
K_{n i}=K_{i n}=-\frac{\phi^{\prime \prime}}{\phi}, & i=1, \ldots, n-1, \\
K_{i j}=-\frac{\left(\phi^{\prime}\right)^{2}-1}{\phi^{2}}, & i, j=1, \ldots, n-1, i \neq j .
\end{array}
$$

The Ricci curvature is then calculated as

$$
\begin{aligned}
& \operatorname{Ric}\left(F_{i}\right)=-\left((n-2) \frac{\left(\phi^{\prime}\right)^{2}-1}{\phi^{2}}+\frac{\phi^{\prime \prime}}{\phi}\right) F_{i}, \quad i<n, \\
& \operatorname{Ric}\left(F_{n}\right)=-(n-1) \frac{\phi^{\prime \prime}}{\phi} F_{n},
\end{aligned}
$$

We denote by $S(r)$ and $B(r), r>0$, the geodesic sphere and ball with center 0 and radius $r$, respectively. The volume of $B(r)$ and the area of $S(r)$ are calculated from

$$
|B(r)|=\omega_{n} \int_{0}^{r} \phi(s)^{n-1} d s, \quad|S(r)|=\omega_{n} \phi(r)^{n-1},
$$

where $\omega_{n}$ is the area of the standard unit sphere $\mathbb{S}^{n-1}$.

In the particular case of Euclidean space, $\phi(r)=r$, while for hyperbolic space, $\phi(r)=\sinh r$. Typical examples are $\phi(r)=r+\beta r^{\alpha}$ for some constants $\alpha \geq 2$ and $\beta>0$, and

$$
\phi(r)=\sum_{k=0}^{n} \frac{r^{(2 k+1)}}{k !}
$$

for some $n \in \mathbb{R}^{n}$, which serve as an interpolation space between Euclidean space and hyperbolic space. Their sectional curvature is non-positive and Ricci curvature is bounded below by a negative constant.

Rotationally symmetric manifolds are important in the sense that they serve as models for more general problems (see, for instance, [34] for the study of manifolds having a pole, [47] for Brownian motion and probability aspects). However, we have not found in the literature a clean if and only if condition for having suitable heat kernel upper bounds (see [40] for examples in this direction).

We define the ratios

$$
f_{1}(r)=\frac{\phi^{\prime \prime}(r)}{\phi(r)}, \quad f_{2}(r)=(n-2) \frac{\left(\phi^{\prime}\right)^{2}-1}{\phi^{2}}+\frac{\phi^{\prime \prime}}{\phi} .
$$

Recalling Proposition 3.3, we are in a good situation if, for instance: both $f_{1}, f_{2}$ are uniformly upper-bounded and the volume control (3.7) holds.

One may compare the volume of any ball to the volume of a ball centered at the origin using (3.6). First, if $1 \geq 2 d_{M}(x, 0)$, then

$$
|B(0,1)| \leq\left|B\left(0,1-d_{M}(x, 0)\right)\right| \frac{\left|B_{\beta}(1)\right|}{\left|B_{\beta}\left(1-d_{M}(x, 0)\right)\right|} \leq C_{n}|B(x, 1)| .
$$


On the other hand, if $1<2 d_{M}(x, 0)$, then

$$
|B(0,1)| \leq|B(0,1 / 4)| \frac{\left|B_{\beta}(1)\right|}{\left|B_{\beta}(1 / 4)\right|} \leq C_{n}\left|B\left(x, d_{M}(x, 0)+1 / 4\right)\right|,
$$

and if we use comparison again,

$$
\begin{aligned}
\left|B\left(x, d_{M}(x, 0)+1 / 4\right)\right| & \leq|B(x, 1 / 4)| \frac{\left|B_{\beta}\left(d_{M}(x, 0)+1 / 4\right)\right|}{\left|B_{\beta}(1 / 4)\right|} \\
& \leq C_{n}|B(x, 1)|\left(1+d_{M}(x, 0)\right)^{n}
\end{aligned}
$$

Therefore

$$
\frac{1}{|B(x, 1)|} \leq \frac{1}{|B(0,1)|} C_{n}\left(1+d_{M}(x, 0)\right)^{n}
$$

so (3.7) is fulfilled. Summarizing we have obtained:

Proposition 3.5. Let $M$ be a (noncompact) rotationally symmetric manifold with metric (3.11), with $\phi$ as given at the beginning of the section. Then $M$ satisfies condition (III) in Theorem 3.2 if $f_{1}(r), f_{2}(r)$ are uniformly bounded from above for $r \in[0, \infty)$.

Remark 3.6. Under conditions on the sectional curvature, estimates have been obtained also on the Schrodinger operator $e^{i t \Delta_{M}}$ in [8].

Remark 3.7. In the case of rotationally symmetric manifolds there is an equivalent way to write the extension problem: we define the weight

$$
w(r)=\left(\frac{r}{\phi(r)}\right)^{(n-1) / 2}
$$

that provides an isometry between $L^{2}(M)$ and $L^{2}\left(\mathbb{R}^{n}\right)$. Then one has the conjugation formula

$$
\Delta_{M} h=w L\left(w^{-1} h\right)
$$

with

$$
L=\partial_{r r}-\frac{n-1}{r} \partial_{r}+\frac{1}{\phi^{2}} \Delta_{\mathbb{S}^{n-1}}-V,
$$

and $V$ the radial function (see for instance [8]),

$$
V(r)=\frac{n-1}{2} \frac{\phi^{\prime \prime}}{\phi}-\frac{(n-1)(n-3)}{4}\left(\left(\frac{\phi^{\prime}}{\phi}\right)^{2}-\frac{1}{r^{2}}\right) .
$$

On one hand we obtain, if the operators are positive (which is the case if for instance $V$ is nonnegative),

$$
\left(-\Delta_{M}\right)^{\gamma} h=w(L)^{\gamma}\left(w^{-1} h\right) .
$$


On the other hand, by the change of function $v(r, \omega, y)=w(r) u(r, \omega, y)$, the system (2.11) is transformed into

$$
\left\{\begin{aligned}
\partial_{y y} u+\frac{a}{y} \partial_{y} u+L u(x, y) & =0 & & \text { for }(x, y) \in \mathbb{R}^{n} \times \mathbb{R}_{+}, \\
u(x, 0) & =w^{-1}(|x|) f(x) & & \text { for } x \in \mathbb{R}^{n} .
\end{aligned}\right.
$$

We apply again the results of [62], this time for the operator $L$ acting on functions on $\mathbb{R}^{n}$, and obtain the existence of the solution of (3.13) (and implicitly of (1.1)), together with the limit

$$
(L)^{\gamma}\left(w^{-1} f\right)=-d_{\gamma} \lim _{y \rightarrow 0} y^{a} \partial_{y} u,
$$

In view of the definition of $u$ we get (1.2):

$$
-d_{\gamma} \lim _{y \rightarrow 0} y^{a} \partial_{y} v=-w d_{\gamma} \lim _{y \rightarrow 0} y^{a} \partial_{y} u=w(L)^{\gamma}\left(w^{-1} f\right)=\left(-\Delta_{M}\right)^{\gamma} f .
$$

Finally, taking Fourier transform on $\mathbb{R}^{n},(3.13)$ gets transformed into

$$
\partial_{y y} \hat{u}+\frac{a}{y} \partial_{y} \hat{u}-\lambda^{2} \hat{u}-\hat{V} \star \hat{u}=0
$$

which, a priori, may not be explicitly solved, but it may give further information.

Rotationally symmetric manifolds are toy models of warped products and one could continue this study further. We refer the reader to [35].

3.3.4. Manifolds with ends. Let us consider first the case that $M$ is topologically of the form $X \times(0, \infty)$, where the manifold $X$ need not be compact; note that the extension to several cusps is straightforward. Assume that $X$ has dimension $N \geq 2$ and carries a metric $g_{X}$. Define the metric on $M$ as

$$
g_{M}=\gamma(x, r)\left(g_{X}+d r^{2}\right), \quad x \in M, r \in(0, \infty) .
$$

In addition, we assume that $M$ is approximately hyperbolic in the sense that $\gamma$ is a positive $\mathcal{C}^{\infty}$ function which satisfies

$$
c^{-1} r^{-2} \leq \gamma(x, r) \leq c r^{-2}, \quad\left|\partial_{r} \gamma\right| \leq c r^{-3} \quad \text { on } M
$$

for some $c>0$. The Laplace-Beltrami operator is written as

$$
\Delta_{M}=\gamma^{-(N+1) / 2} \operatorname{div}_{x}\left(\gamma^{(N-1) / 2} \nabla_{x}\right)+\gamma^{-(N+1) / 2} \partial_{r}\left(\gamma^{(N-1) / 2} \partial_{r}\right) .
$$

It is shown in [23] (see also [54]) that if the heat kernel of $X$ satisfies the bound

$$
0 \leq p_{t}\left(x, x^{\prime}\right) \leq c t^{-N / 2}, \quad \text { for all } 0<t \leq 1, x, x^{\prime} \in X,
$$

which is the case when $X$ is compact, then the heat kernel of $M$ satisfies

$$
0 \leq p_{t}\left(m, m^{\prime}\right) \leq c_{\delta}(1+r)^{N / 2}\left(1+r^{\prime}\right)^{N / 2} t^{-(N+1) / 2} e^{\left(2 \delta-\lambda_{1}\right) t} e^{-\frac{d_{M}\left(m, m^{\prime}\right)^{2}}{4(1+\delta) t}}
$$


for all $0<t<\infty, m, m^{\prime} \in M$, where $\lambda_{1}$ is the bottom of the spectrum of $-\Delta_{M}$. Since one can get as for (3.12) the estimate

$$
\frac{1}{|B(x, \sqrt{t})|} \leq \frac{1}{|B(0, \sqrt{t})|} C_{n}\left(1+\frac{d_{M}(x, 0)}{\sqrt{t}}\right)^{n},
$$

plugging it into (3.5) hat we can satisfy condition (III) in Theorem 3.2.

More generally, one may consider weighted complete manifolds of the form

$$
M=M_{1} \# \cdots \# M_{k}
$$

that is, manifolds that are the connected sum of a finite number of manifolds $M_{i}$, $1 \leq i \leq k$. More precisely, this means that $M$ is the disjoint union $M=K \cup$ $E_{1} \cup \cdots \cup E_{k}$, where $K$ is a compact with smooth boundary (we refer to it as the central part) and each $E_{i}$ is isometric to the complement of a compact set $K_{i}$ with smooth boundary in $M_{i}$. If $M$ is weighted then we assume that the $M_{i}$ 's are weighted. The weight on $M$ and the weight on $M_{i}$ coincide on $E_{i}$ (with the obvious identification). The goal of [39] is to study heat kernel bounds for $M$ from the bounds on each $M_{i}$ through a gluing technique. In order to keep the presentation simple, we will not state their theorem in full generality, but explain the model cases that inspire it.

For an integer $m \in[1, N]$ we define the manifold $\mathcal{R}^{m}$ by

$$
\mathcal{R}^{1}=\mathbb{R}_{+} \times \mathbb{S}^{N-1}, \quad \mathcal{R}^{m}=\mathbb{R}^{m} \times \mathbb{S}^{N-m}, m \geq 2 .
$$

The manifold $\mathcal{R}^{m}$ has topological dimension $N$ but its "dimension at infinity" is $m$ in the sense that $V(x, r) \sim r^{m}$ for $r \geq 1$. This enables to consider finite connected sums $M=\mathcal{R}^{N_{1}} \# \cdots \# \mathcal{R}^{N_{k}}$, for fixed $M$ and $k$ integers $N_{1}, N_{2}, \ldots, N_{k} \in[1, N]$.

We assume that all ends of $M$ are non-parabolic, i.e., each $N_{i}>2$, and set

$$
n:=\min _{1 \leq i \leq k} N_{i}(>2)
$$

Let $K$ be the central part of $M$ and $E_{1}, \ldots, E_{k}$ be the ends of $M$ so that $E_{i}$ is isometric to the complement of a compact set in $\mathcal{R}^{N_{i}}$. With some abuse of notation, we write $E_{i}=\mathcal{R}^{N_{i}} \backslash K$. For any point in $x \in M$, set

$$
|x|:=\sup _{x^{\prime} \in K} d\left(x, x^{\prime}\right) .
$$

Observe that since $K$ has non-empty interior, $x$ is separated from 0 on $M$ and $|x| \sim 1+d(x, K)$.

For instance, let $k=2$ (i.e., $M$ has two ends). Set $M=\mathcal{R}^{n} \# \mathcal{R}^{m}, 2<n \leq m$. Assume that $x \in \mathcal{R}^{n} \backslash K, x^{\prime} \in \mathcal{R}^{m} \backslash K, t \geq t_{0}$. Then we have the heat kernel bound

$$
0 \leq p_{t}\left(x, x^{\prime}\right) \leq C\left(\frac{1}{t^{m / 2}|x|^{n-2}}+\frac{1}{t^{n / 2}\left|x^{\prime}\right|^{m-2}}\right) e^{-c \frac{d^{2}}{t}},
$$

which is enough for our purposes. 


\subsection{Other frameworks}

One may also consider the construction of the fractional Laplacian on metric graphs. Note that heat kernel estimates are also valid for the Laplacian on graphs, see for instance the recent studies [28], [48] and references therein as [66].

Finally, it would be interesting to construct real and complex powers of the complex Laplacian on Kähler manifolds.

\section{References}

[1] Abramowitz, M. And Stegun, I. A.: Handbook of mathematical functions with formulas, graphs, and mathematical tables. National Bureau of Standards Applied Mathematics Series 55, US Government Printing Office, Washington, DC, 1964.

[2] Anker, J. P.: $L_{p}$ Fourier multipliers on Riemannian symmetric spaces of the noncompact type. Ann. of Math. (2) 132 (1990), no. 3, 597-628.

[3] Anker, J.P., Damek, E. And Yacoub, C.: Spherical analysis on harmonic AN groups. Ann. Scuola Norm. Sup. Pisa Cl. Sci. (4) 23 (1996), no. 4, 643-679.

[4] Anker, J. P. And Ji, L.: Heat kernel and Green function estimates on noncompact symmetric spaces. Geom. Funct. Anal. 9 (1999), no. 6, 1035-1091.

[5] Anker, J.P., Pierfelice, V. And Vallarino, M.: Schrödinger equations on Damek-Ricci spaces. Comm. Partial Differential Equations 36 (2011), no. 6, 976-997.

[6] Anker, J.P. And Ostellari, P.: The heat kernel on noncompact symmetric spaces. In Lie groups and symmetric spaces, 27-46. Amer. Math. Soc. Transl. Ser. 2, 210, Amer. Math. Soc., Providence, RI, 2003.

[7] BanicA, V.: The nonlinear Schrödinger equation on hyperbolic space. Comm. Partial Differential Equations 32 (2007), no. 10-12, 1643-1677.

[8] Banica, V. AND Duyckaerts, T.: Weighted Strichartz estimates for radial Schrödinger equation on noncompact manifolds. Dyn. Partial Differ. Equ. 4 (2007), no. $4,335-359$.

[9] Bowditch, B. H.: Geometrical finiteness for hyperbolic groups. J. Funct. Anal. 113 (1993), no. 2, 245-317.

[10] Berenstein, C. And Casadio Tarabusi, E.: Inversion formulas for the $k$ dimensional Radon transform in real hyperbolic spaces. Duke Math. J. 62 (1991), no. $3,613-631$.

[11] Caffarelli, L. A. And Silvestre, L. E.: An extension problem related to the fractional laplacian. Comm. Partial Differential Equations 32 (2007), no. 7-9, 1245-1260.

[12] Cabré, X. And Sire, Y.: Non-linear equations for fractional Laplacians I: Regularity, maximum principles, and Hamiltoniam estimates. Ann. Inst. H. Poincaré Anal. Non Linéaire 31 (2014), no. 1, 23-53.

[13] Castorina, D., Fabbri, I., Mancini, G. and Sandeep, K.: Hardy-Sobolev extremals, hyperbolic symmetry and scalar curvature equations. J. Differential Equations 246 (2009), no. 3, 1187-1206.

[14] Cheeger, J. And Yau, S. T.: A lower bound for the heat kernel. Comm. Pure Appl. Math. 34 (1981), no. 4, 465-480. 
[15] Chang, A. And Gonzalez, M. D. M.: Fractional Laplacian in conformal geometry. Adv. Math. 226 (2011), no. 2, 1410-1432.

[16] Cheng, S. Y., Li, P. And Yau, S. T.: On the upper estimate of the heat kernel of a complete Riemannian manifold. Amer. J. Math. 103 (1981), no. 5, 1021-1063.

[17] Cohen, S. And Lifshits, M. A.: Stationary Gaussian random fields on hyperbolic spaces and on Euclidean spheres. ESAIM Probab. Stat. 16 (2012), 165-221.

[18] Cotsiolis, A. And Tavoularis, N. K.: Best constants for Sobolev inequalities for higher order fractional derivatives. J. Math. Anal. Appl. 295 (2004), no. 1, 225-236.

[19] Damek, E. And Ricci, F.: Harmonic analysis on solvable extensions of $H$-type groups. J. Geom. Anal. 2 (1992), no. 3, 213-248.

[20] Davies, E. B.: Heat kernels and spectral theory. Cambridge Tracts in Mathematics 92, Cambridge University Press, Cambridge, 1990.

[21] Davies, E. B.: Gaussian upper bounds for the heat kernels of some second-order operators on Riemannian manifolds. J. Funct. Anal. 80 (1988), no. 1, 16-32.

[22] Davies, E. B. And Mandouvalos, N.: Heat kernel bounds on hyperbolic space and Kleinian groups. Proc. London Math. Soc. (3) 57 (1988), no. 1, 182-208.

[23] Davies, E. B. and Mandouvalos, N.: Heat kernel bounds on manifolds with cusps. J. Funct. Anal. 75 (1987), no. 2, 311-322.

[24] Debiard, A., Gaveau, B. and Mazet, E.: Théorèmes de comparaison en géométrie riemannienne. Publ. Res. Inst. Math. Sci. 12 (1976/77), no. 2, 391-425.

[25] Fabes, E. B., Kenig, C. E. and Serapioni, R. P.: The local regularity of solutions of degenerate elliptic equations. Comm. Partial Differential Equations 7 (1982), no. 1, $77-116$.

[26] Fabes, E. B., Kenig, C. E. And Jerison, D.: Boundary behavior of solutions to degenerate elliptic equations. In Conference on harmonic analysis in honor of Antoni Zygmund, Vol. I, II (Chicago, Ill., 1981), 577-589. Wadsworth Math. Ser., Wadsworth, Belmont, CA, 1983.

[27] Folland, G. B.: Subelliptic estimates and function spaces on nilpotent Lie groups. Ark. Mat. 13 (1975), no. 2, 161-207.

[28] Frank, R. L. And Kovařík, H.: Heat kernels of metric trees and applications. SIAM J. Math. Anal. 45 (2013), no. 3, 1027-1046.

[29] Gelfand, I. M., Gindikin, S. G. and Graev, I. M.: Selected topics in integral geometry. Translations of Mathematical Monographs 220, American Mathematical Society, Providence, RI, 2003.

[30] González, M.d. M., Mazzeo, R. And Sire, Y.: Singular solutions of fractional order conformal laplacians. J. Geom. Anal. 22 (2012), no. 3, 845-863.

[31] GonzÁlez, M. D. M., AND Qing, J.: Fractional conformal Laplacians and fractional Yamabe problems. Anal. PDE 6 (2013), no. 7, 1535-1576.

[32] GonzÁlez, M. D. M., SÁez, M. and Sire, Y.: Layer solutions for the fractional Laplacian on hyperbolic space: existence, uniqueness and qualitative properties. Ann. Mat. Pura Appl. (4) 193 (2014), no. 6, 1823-1850.

[33] Graham, C. R. And Zworski, M.: Scattering matrix in conformal geometry. Invent. Math. 152 (2003), no. 1, 89-118.

[34] Greene, R. E. And Wu, H.: Function theory on manifolds which possess a pole. Lecture Notes in Mathematics 699, Springer, Berlin, 1979. 
[35] Grigor'yan, A.: Heat kernels on weighted manifolds and applications. In The ubiquitous heat kernel, 93-191. Contemp. Math. 398, American Mathematical Society, Providence, RI, 2006.

[36] Grigor'Yan, A.: Analytic and geometric background of recurrence and nonexplosion of the Brownian motion on Riemannian manifolds. Bull. Amer. Math. Soc. (N.S.) 36 (1999), no. 2, 135-249.

[37] Grigor'YAn, A.: Gaussian upper bounds for the heat kernel and for its derivatives on a Riemannian manifold. In Classical and modern potential theory and applications (Chateau de Bonas, 1993), 237-252. NATO Adv. Sci. Inst. Ser. C Math. Phys. Sci. 430, Kluwer Acad. Publ., Dordrecht, 1994.

[38] Grigor'yan, A.: Estimates of heat kernels on Riemannian manifolds. In Spectral theory and geometry (Edinburgh, 1998), 140-225. London Math. Soc. Lecture Note Ser. 273, Cambridge Univ. Press, Cambridge, 1999.

[39] Grigor'yan, A. And Saloff-Coste, L.: Heat kernel on manifolds with ends. Ann. Inst. Fourier (Grenoble) 59 (2009), no. 5, 1917-1997.

[40] Grigor'yan, A. And Saloff-Coste, L.: Stability results for Harnack inequalities. Ann. Inst. Fourier (Grenoble) 55 (2005), no. 3, 825-890.

[41] Grunau, H. C. Ould Ahmedou, M. and Reichel, W.: The Paneitz equation in hyperbolic space. Ann. Inst. H. Poincaré Anal. Non Linéaire 25 (2008), no. 5, $847-864$.

[42] Guillarmou, C. And Mazzeo, R.: Resolvent of the Laplacian on geometrically finite hyperbolic manifolds. Invent. Math. 187 (2012), no. 1, 99-144.

[43] Helgason, S: The totally-geodesic Radon transform on constant curvature spaces. In Integral geometry and tomography (Arcata, CA, 1989), 141-149. Contemp. Math. 113, Amererican Mathematical Society, Providence, RI, 1990.

[44] Helgason, S: Geometric analysis on symmetric spaces. Mathematical Surveys and Monographs 39, American Mathematical Society, Providence, RI, 2008.

[45] Helgason, S: Groups and geometric analysis. Integral geometry, invariant differential operators, and spherical functions. Mathematical Surveys and Monographs 83, American Mathematical Society, Providence, RI, 2000.

[46] Helgason, S: Differential geometry, Lie groups, and symmetric spaces. Graduate Studies in Mathematics 34, American Mathematical Society, Providence, RI, 2001.

[47] Hsu, E.: Stochastic analysis on manifolds. Graduate Studies in Mathematics 38, American Mathematical Society, Providence, RI, 2002.

[48] Keller, M., Lenz, D. And Wojciechowski, R.: Volume growth, spectrum and schochastic completeness of infinite graphs. Math. Z. 274 (2013), no. 3-4, 905-932.

[49] Li, P. And YAU, S. T.: On the parabolic kernel of the Schrödinger operator. Acta Math. 156 (1986), no. 1, 153-201.

[50] Liu, G.: Sharp higher-order Sobolev inequalities in the hyperbolic space $\mathbb{H}^{n}$. Calc. Var. Partial Differential Equations 47 (2013), no. 3-4, 567-588.

[51] Mancini, G. And Sandeep, K.: On a semilinear elliptic equation in $\mathbb{H}^{n}$. Ann. Sc. Norm. Super. Pisa Cl. Sci. (5) 7 (2008), no. 4, 635-671.

[52] Mancini, G. And Sandeep, K.: Extremals for Sobolev and Moser inequalities in hyperbolic space. Milan J. Math. 79 (2011), no. 1, 273-283.

[53] Mazzeo, R. And Melrose, R.: Meromorphic extension of the resolvent on complete spaces with asymptotically constant negative curvature. J. Funct. Anal. 75 (1987), no. $2,260-310$. 
[54] Müller, W.: Spectral theory for Riemannian manifolds with cusps and a related trace formula. Math. Nachr. 111 (1983), 197-288.

[55] Perry, P.: Meromorphic continuation of the resolvent for Kleinian groups. In Spectral problems in geometry and arithmetic (Iowa City, IA, 1997), 123-147. Contemp. Math. 237, American Mathematical Society, Providence, RI, 1999.

[56] Petersen, P.: Riemannian geometry. Graduate Texts in Mathematics 171, Springer-Verlag, New York, 1998.

[57] Ratcliffe, J.: Foundations of hyperbolic manifolds. Graduate Texts in Mathematics 149, Springer-Verlag, New York, 1994.

[58] Roncal, L. And Stinga, P. R.: Fractional Laplacian on the torus. To appear in Commun. Contemp. Math.

[59] Rudin, W.: Functional analysis. McGraw-Hill Series in Higher Mathematics, McGraw-Hill, New York-Ds̈seldorf-Johannesburg, 1973.

[60] Saloff-Coste, L.: The heat kernel and its estimates. In Probabilistic approach to geometry, 405-436. Adv. Stud. Pure Math. 57, Math. Soc. Japan, Tokyo, 2010.

[61] Silvestre, L.: Regularity of the obstacle problem for a fractional power of the Laplace operator. Comm. Pure Appl. Math. 60 (2007), no. 1, 67-112.

[62] Stinga, P.R. And Torrea, J. L.: Extension problem and Harnack's inequality for some fractional operators. Commun. Partial Differ. Equations 35 (2010), no. 11, $2092-2122$.

[63] Tataru, D.: Strichartz estimates in the hyperbolic space and global existence for the semilinear wave equation. Trans. Amer. Math. Soc. 353 (2001), no. 2, 795-807.

[64] Terras, A.: Harmonic analysis on symmetric spaces and applications. I. SpringerVerlag, New York, 1985.

[65] Triebel, H.: Theory of function spaces. II. Monographs in Mathematics 84, Birkhäuser Verlag, Basel, 1992.

[66] Urakawa, H.: Heat kernel and Green kernel comparison theorems for infinite graphs. J. Funct. Anal. 146 (1997), no. 1, 206-235.

[67] Valdinoci, E.: From the long jump random walk to the fractional Laplacian. Bol. Soc. Esp. Mat. Apl. SëMA 49 (2009), 33-44.

[68] Varopoulos, N.: Semi-groupes d'opérateurs sur les espaces $L^{p}$. C.R. Acad. Sci. Paris Sér. I Math. 301 (1985), no. 19, 865-868.

[69] YosidA, K.: Functional analysis. Die Grundlehren der Mathematischen Wissenschaften 123, Academic Press, New York; Springer-Verlag, Berlin, 1965.

Received December 17, 2012; revised October 9, 2013.

VALERIA BANICA: Laboratoire Analyse et probabilités (EA 2172), Université d'Evry, 23 Bd. de France, 91037 Evry, France.

E-mail: valeria.banica@univ-evry.fr

The first author is partially supported by the French ANR projects R.A.S. ANR-08-JCJC0124-01 and SchEq ANR-12-JS01-0005-01. The second author is supported by grants MTM201127739-C04-01 (Spain), and 2009SGR345 (Catalunya). The third author is supported by grants Fondecyt regular 1110048 and proyecto Anillo ACT-125, CAPDE. 
María del Mar GonzÁlez: Universitat Politècnica de Catalunya, ETSEIB-MA1, Av. Diagonal 647, 08028 Barcelona, Spain.

E-mail: mar.gonzalez@upc.edu

Mariel SÁez: Pontificia Universidad Católica de Chile, Avda. Vicuña Mackenna 4860, Macul, 6904441, Santiago, Chile.

E-mail: mariel@mat.puc.cl 